\title{
Identifying rotational Radon transforms
}

\author{
ÁrpÁD KurusA*
}

\begin{abstract}
We show classes of test functions so that dilational and rotational invariances of the image $\mathrm{R}_{\mathcal{S}, \mu} f$ of such a test function $f$ determines dilational and rotational invariances of rotational Radon transform $\mathrm{R}_{\mathcal{S}, \mu}$. Then we determine the defining flower $\mathcal{S}$ and weight $\mu$ of a conformal Radon transform $\mathrm{R}_{\mathcal{S}, \mu}$ in terms of the image $\mathrm{R}_{\mathcal{S}, \mu} f$ of an unknown function that is a sum of an $\mathrm{L}^{2}$-function and finitely many Dirac distributions if the flower $\mathcal{S}$ is not selftangent.
\end{abstract}

\section{Introduction}

Let $\mathcal{S}_{\omega}$ be a set of hypersurfaces $S_{\omega, t}$ in $\mathbb{R}^{n}$ so that $\omega \in \mathbb{S}^{n-1}$ and $t \in[0, \infty)$. The Radon transform $\mathrm{R}_{\mathcal{S}, \mu}$ of functions $f: \mathbb{R}^{n} \rightarrow \mathbb{R}$ integrable on each $S_{\omega, t}$ is defined by

$$
\mathrm{R}_{\mathcal{S}, \mu} f(\omega, t)=\int_{S_{\omega, t}} f(\mathbf{x}) \mu_{\omega, t}(\mathbf{x}) \mathrm{d} \mathbf{x}
$$

where $\mathrm{d} \mathbf{x}$ is the natural surface measure on $S_{\omega, t}$ and $\mu_{\omega, t}$ is a strictly positive continuous function on $S_{\omega, t}$ that depends continuously on $\omega$ and $t$. In this definition, the hypersurfaces $S_{\omega, t}$ are called the petals, the set $\mathcal{S}=\bigcup_{\omega \in \mathbb{S}^{n-1}} \mathcal{S}_{\omega}$ of them is called flower and $\mu_{\omega, t}$ is called the weight on the petal $S_{\omega, t}$.

In terms of this definition the "classic" Radon transform $\mathrm{R}_{\mathcal{H}, \mu}$ is defined by the flower $\mathcal{H}=\left\{H_{\omega, t}: \omega \in \mathbb{S}^{n-1}, t \in[0, \infty)\right\}$ of the petals $H_{\omega, t}=\{\mathbf{x}:\langle\mathbf{x}, \omega\rangle=t\}$ with weight $\mu_{\omega, t} \equiv 1$.

AMS Subject Classification (2000): 44A12.

Key words and phrases: invariant Radon transform, identification of Radon transform, rotational Radon transform, conformal Radon transform.

* Supported by the European Union and co-funded by the European Social Fund under the project "Telemedicine-focused research activities on the field of Matematics, Informatics and Medical sciences" of project number "TÁMOP-4.2.2.A-11/1/KONV-2012-0073".

Period.Math. Hungar., 67:2 (2013), 187-209.

(c) Á. Kurusa 
The problem considered in this article is to identify an a priori unknown Radon transform $\mathrm{R}_{\mathcal{S}, \mu}$ or the properties of its flower and/or weight by means of the known images $\mathrm{R}_{\mathcal{S}, \mu} f$ of (partially) (un)known test functions $f$.

In the special case where the petals are total geodesics of a certain Riemannian manifold Mukhometov proves in [9] and [10] that $\mathrm{R}_{\mathcal{S}, 1} 1$ determines $\mathcal{S}$.

Considering the attenuated Radon transform $\mathrm{R}_{\mathcal{H}, \mu}$ which was arisen in single photon emission computed tomography Natterer shows in [11] and [12] that the weight $\mu$ can be computed up to a multiplicative function in the support of the test function $f$ if $f$ is known to be a finite sum of Dirac measures. Hertle proves in [5] that for constantly attenuated Radon transforms $\mathrm{R}_{\mathcal{H}, \mu}$ the map $(f, \mu) \mapsto \mathrm{R}_{\mathcal{H}, \mu} f$ is injective on the set of the compactly supported, not radial distributions $f$. Solmon shows in [16] that the weight $\mu$ of an exponential Radon transform (which is a synonym for constantly attenuated Radon transforms) $\mathrm{R}_{\mathcal{H}, \mu}$ can be computed from $\mathrm{R}_{\mathcal{H}, \mu} f$ if and only if the compactly supported distribution $f$ is not radial.

For generalized Radon transforms J. Boman justifies in [1] that if $\mathrm{R}_{\mathcal{H}, \mu} f=$ $\mathrm{R}_{\mathcal{H}, \nu} g$ for the compactly supported measures $f, g$, and $f$ is a sum of an $\mathrm{L}^{2}$-function and a finite sum $f_{0}$ of Dirac measures, then $g$ is also a sum of an $\mathrm{L}^{2}$-function and a finite sum $g_{0}$ of Dirac measures and $f_{0} \mu \equiv g_{0} \nu$.

In this article, first we describe the class of Radon transforms we are studying then present some preliminary results on appropriate flowers and on the dual Radon transforms.

In Section 3 we show classes of test functions so that dilational and rotational invariances of the image $\mathrm{R}_{\mathcal{S}, \mu} f$ of such a test function $f$ determine dilational and rotational properties of the defining flower $\mathcal{S}$ and weight $\mu$.

Finally in Section 4 we determine the defining flower $\mathcal{S}$ and weight $\mu$ of any conformal Radon transform $\mathrm{R}_{\mathcal{S}, \mu}$ in terms of the image $\mathrm{R}_{\mathcal{S}, \mu} f$ of an unknown function that is a sum of an $\mathrm{L}^{2}$-function and finitely many Dirac distributions if the flower $\mathcal{S}$ has no two tangent petals.

\section{Preliminaries}

We call a flower $\mathcal{S}$ in $\mathbb{R}^{n}(2 \leq n \in \mathbb{N})$

- symmetric if $\mathbf{x} \in S_{\omega, t}$ is equivalent to $2\langle\mathbf{x}, \omega\rangle \omega-\mathbf{x} \in S_{\omega, t}$, i.e., the petals $S_{\omega, t}$ are invariant against the reflection onto the straight line through the origin with direction $\omega$,

- rotational if $S_{\Phi \omega, t}=\Phi\left(S_{\omega, t}\right)$ for any rotation $\Phi \in S O(n)$, i.e., any rotation around the origin rotates a petal into a petal with the same second parameter and rotated first parameter, and 
- dilational if $\bar{t} S_{\omega, t}=t S_{\omega, \bar{t}}$ for all $t, \bar{t}>0$, i.e., any homogeneous dilation with the origin as homothetic centre takes a petal into a petal with the same first parameter and dilated second parameter.

Observe, that in dimensions $n \geq 3$ the petals $S_{\omega, t}$ of a rotational flower $\mathcal{S}$ are rotationally symmetric around the straight line through the origin with direction $\omega$ and therefore they are also symmetric. If a flower is rotational and dilational, then we call it conformal.

A weight $\mu$ on a flower $\mathcal{S}$ is called

- symmetric if $\mathcal{S}$ is symmetric and $\mu_{\omega, t}(\mathbf{x})=\mu_{\omega, t}(2\langle\mathbf{x}, \omega\rangle \omega-\mathbf{x})$ for all $\mathbf{x} \in S_{\omega, t}$,

- rotational if $\mathcal{S}$ is rotational and $\mu_{\Phi \omega, t}(\Phi \mathbf{x})=\mu_{\omega, t}(\mathbf{x})$ for all $\Phi \in S O(n)$, $\omega \in \mathbb{S}^{n-1}, t \in \mathbb{R}$ and $\mathbf{x} \in S_{\omega, t}$, and

- dilational if $\mathcal{S}$ is dilational and $\mu_{\omega, p t}(p \mathbf{x})=\mu_{\omega, t}(\mathbf{x})$ for all $p>0$.

If a weight is rotational and dilational, then it is called conformal. Observe, that a rotational weight $\mu$ has necessarily the form

$$
\mu_{\omega, r}(\mathbf{x})= \begin{cases}\bar{\mu}_{r}\left(|\mathbf{x}| \frac{\left\langle\mathbf{x}, \omega^{\perp}\right\rangle}{\left\langle\mathbf{x}, \omega^{\perp}\right\rangle}\right), & \text { if } n=2, \\ \bar{\mu}_{r}(|\mathbf{x}|), & \text { if } n>2 \text { or it is symmetric in } n=2\end{cases}
$$

for a suitable function $\bar{\mu}: \mathbb{R}_{+} \times \mathbb{R} \rightarrow \mathbb{R}$.

We call a Radon transform of form (1.1) symmetric, rotational, dilational or conformal if both the flower and the weight are symmetric, rotational, dilational or conformal, respectively. To mention some examples, the classic Radon transform is symmetric conformal, while the exponential Radon transform is symmetric and rotational but not dilational.

A piecewise differentiable image $c_{r}$ of an interval in the plane is called an inner (outer) nice curve if in a coordinate system

(1) $c_{r}$ has a unique point $P_{r}$ farthest from (closest to) the origin, so that $P_{r}=$ $(r, 0)$,

(2) each point $c_{r}^{+}(\varrho)$ of the curve $c_{r}$ in the closed upper halfplane is uniquely determined by its distance $\varrho=\left|c_{r}^{+}(\varrho)\right| \geq 0$ to the origin,

(3) each point $c_{r}^{-}(\varrho)$ of the curve $c_{r}$ in the closed downward halfplane is uniquely determined by its distance $\varrho=\left|c_{r}^{-}(\varrho)\right| \geq 0$ to the origin, and

(4) the signed angle $\varphi_{r}^{ \pm}(\varrho)$ of $c_{r}^{ \pm}(\varrho)$ to the $x$-axis as a function is such that $\varphi_{r}^{ \pm}(\varrho) / \sqrt{|r-\varrho|}$ is piecewise $\mathrm{C}^{2}$ on $\operatorname{supp} \varphi_{r}^{ \pm}=\left(\inf _{\exists c_{r}^{ \pm}(\varrho)} \varrho, r\right]\left(\operatorname{supp} \varphi_{r}^{ \pm}=\right.$ $\left.\left[r, \sup _{\exists c_{r}^{ \pm}(\varrho)} \varrho\right)\right)$.

We call a nice curve symmetric ${ }^{(2: 1)}$ if the curves $c_{r}^{ \pm}$are the reflection of each other with respect to the $x$-axis. If this happens, we define $\varphi_{r}(\varrho):= \pm \varphi_{r}^{ \pm}(\varrho)$.

$\overline{(2: 1)}$ This corresponds to the symmetry term defined at the beginning of this section.

Period.Math. Hungar., 67:2 (2013), 187-209.

(c) Á. Kurusa 
A surface in $\mathbb{R}^{n}$ is called inner (outer) nice, if it is obtained by spinning such a symmetric inner (outer) nice plane curve $c_{r}$ around its axis for which

(5) the angle $\varphi_{r}(\varrho)$ of $c_{r}(\varrho)$ to the axis of $c_{r}$ as a function is such that $\varphi_{r}(\varrho) / \sqrt{|r-\varrho|}$ is piecewise $\mathrm{C}^{[(n+2) / 2]}$ on $\operatorname{supp} \varphi_{r}=\left(\inf _{\exists c_{r}(\varrho)} \varrho, r\right]\left(\operatorname{supp} \varphi_{r}=\right.$ $\left.\left[r, \sup _{\exists c_{r}(\varrho)} \varrho\right)\right)$.

Note, that a nice surface in $\mathbb{R}^{2}$ is a symmetric nice curve. Obviously, $\mathcal{H}$ is an outer nice flower, and the set of spheres passing through the origin is an inner nice flower.

According to [7], if a conformal Radon transform $R_{\mathcal{S}, 1}$ is invertible, then the petals of its flower are nice (see [2], [3] for special nice flowers). Based on this result, without further notice in this article we restrict our considerations to rotational Radon transforms with nice flowers, and generally work out the calculations only for inner nice flowers.

On a nice curve $c_{r}$ the arc length measure at $c_{r}(\varrho)$ is $\sqrt{1+\left(\varrho \dot{\varphi}_{r}^{ \pm}(\varrho)\right)^{2}} d \varrho[7]$, therefore a rotational Radon transform in the plane takes the form

$$
\mathrm{R}_{\mathcal{S}, \mu} f\left(\omega_{\alpha}, r\right)=\int_{0}^{r} \sum_{\circ \in \pm} f\left(\varrho \omega_{\alpha+\varphi_{r}^{\circ}(\varrho)}\right) \mu_{\alpha, r}\left(\varrho \omega_{\alpha+\varphi_{r}^{\circ}(\varrho)}\right) \sqrt{1+\varrho^{2}\left(\dot{\varphi}_{r}^{\circ}(\varrho)\right)^{2}} \mathrm{~d} \varrho,
$$

where we have used the practical notation $\omega_{\alpha}$ for the unit vector $(\cos \alpha, \sin \alpha)$.

On a nice surface $S_{r}$ in $\mathbb{R}^{n}(n \geq 3)$ the surface measure at the point $\varrho \omega$ is $\left(\varrho \sin \varphi_{r}(\varrho)\right)^{n-2} \sqrt{1+\varrho^{2} \dot{\varphi}_{r}^{2}(\varrho)} \mathrm{d} \omega \mathrm{d} \varrho[7]$, hence a rotational Radon transform in $\mathbb{R}^{n}$ $(n \geq 3)$ takes the form

$$
\begin{aligned}
& \mathrm{R}_{\mathcal{S}, \mu} f(\bar{\omega}, r) \\
&=\int_{0}^{r} \int_{\mathbb{S}_{\bar{\omega}}^{n-2}} f\left(\varrho \left(\omega \sin \varphi_{r}(\varrho)+\right.\right.\left.\left.\bar{\omega} \cos \varphi_{r}(\varrho)\right)\right) \times \\
& \times \mu_{\bar{\omega}, r}\left(\varrho\left(\omega \sin \varphi_{r}(\varrho)+\bar{\omega} \cos \varphi_{r}(\varrho)\right)\right) \mathrm{d} \omega \times \\
& \times\left(\varrho \sin \varphi_{r}(\varrho)\right)^{n-2} \sqrt{1+\varrho^{2} \dot{\varphi}_{r}^{2}(\varrho)} \mathrm{d} \varrho,
\end{aligned}
$$

where $\bar{\omega}$ is a unit vector and $\mathbb{S}_{\bar{\omega}}^{n-2}$ denotes the $(n-2)$-dimensional unit sphere in the subspace $\mathbb{R}^{n-1}$ perpendicular to $\bar{\omega}$.

Throughout this article for any function $g: \mathbb{R}^{n} \rightarrow \mathbb{R}$ we use the notations $g^{\Phi}(\mathbf{x}):=g(\Phi \mathbf{x})$ and $g_{p}(\mathbf{x}):=g(p \mathbf{x})$ for any $\Phi \in G L(n)$ and for any $0 \neq p \in \mathbb{R}$ respectively. Also the function spaces

$$
\begin{aligned}
\mathrm{L}^{2}\left(\mathbb{R}^{n}, r^{k}\right) & =\left\{f: \mathbb{R}^{n} \rightarrow \mathbb{R}: f(\mathbf{x})|\mathbf{x}|^{k / 2} \in \mathrm{L}^{2}\left(\mathbb{R}^{n}\right)\right\}, \\
\mathrm{L}_{m}^{2}\left(\mathbb{R}^{n}, r^{k}\right) & =\left\{f \in \mathrm{L}^{2}\left(\mathbb{R}^{n}, r^{k}\right):|\mathbf{x}| \leq m \Rightarrow f(\mathbf{x})=0\right\}, \\
\mathrm{L}_{*}^{2}\left(\mathbb{R}^{n}, r^{k}\right) & =\bigcup_{m>0} \mathrm{~L}_{m}^{2}\left(\mathbb{R}^{n}, r^{k}\right),
\end{aligned}
$$

Period.Math. Hungar., $\mathbf{6 7 : 2 ~ ( 2 0 1 3 ) , ~ 1 8 7 - 2 0 9 . ~}$

(c) Á. Kurusa 
where $k \in \mathbb{Z}$ and $m>0$, appear regularly. For the Dirac delta distribution $\delta$ on $\mathbb{R}^{n}$ we use the notation $\delta_{\mathbf{x}}(\mathbf{y}):=\delta(\mathbf{x}-\mathbf{y})$. In the plane $\omega^{\perp}$ is used to denote the unit vector obtained by rotating the unit vector $\omega$ anticlockwise with angle $\pi / 2$, i.e., $\omega_{\varphi}^{\perp}=\omega_{\varphi+\pi / 2}$ with the frequently used notation $\omega_{\alpha}=(\cos \alpha$, $\sin \alpha)$.

We close this section with general observations on the flowers, weights and the Radon transforms they define.

Lemma 2.1. A nice rotational flower in $\mathbb{R}^{n}(n \geq 2)$ is dilational if and only if

$$
\sin ^{n-2} \varphi_{r}^{ \pm}(y r) \sqrt{1+y^{2} r^{2}\left(\dot{\varphi}_{r}^{ \pm}\right)^{2}(y r)}=\sin ^{n-2} \varphi_{1}^{ \pm}(y) \sqrt{1+y^{2}\left(\dot{\varphi}_{1}^{ \pm}\right)^{2}(y)}
$$

for all $y \in[0,1]$ and $r \in[0, \infty)$.

Proof. If the flower is conformal, then clearly $\varphi_{r}^{ \pm}(y r)=\varphi_{1}^{ \pm}(y)$. Differentiation of this by $y$ gives $\dot{\varphi}_{1}^{ \pm}(y)=r \dot{\varphi}_{r}^{ \pm}(y r)$. These two equations prove (2.4).

Now we assume (2.4), and let $\psi(y)=\sin ^{n-2} \varphi_{r}(y r) \sqrt{1+y^{2} r^{2} \dot{\varphi}_{r}^{2}(y r)}$.

Our assumption in dimension 2 implies the independence of $\phi(y):=r \dot{\varphi}_{r}(y r)$ from $r$ that leads to $\varphi_{r}(y r)=\int \phi(y) d y$, whence the statement follows.

In higher dimensions, differentiating the square of $\psi(y)$ with respect to $r$, we obtain

$$
\sin ^{2 n-5} \varphi_{r}(y r)\left(\nu_{r}(y) g_{r}(y)+\lambda_{r}(y) \dot{g}_{r}(y)\right)=\partial_{r} \psi(y) \equiv 0,
$$

where

$$
\begin{aligned}
& g_{r}(y)=\partial_{r}\left(\varphi_{r}(y r)\right)=y \dot{\varphi}_{r}(y r)+\partial_{r} \varphi_{r}(y r), \\
& \nu_{r}(y)=(2 n-4) \cos \varphi_{r}(y r)\left(1+y^{2} r^{2} \dot{\varphi}_{r}^{2}(y r)\right), \\
& \lambda_{r}(y)=\sin \varphi_{r}(y r) 2 r y^{2} \dot{\varphi}_{r}(y r) .
\end{aligned}
$$

By (5) we have a function $h(r, \varrho) \in \mathrm{C}^{[(n+2) / 2]}\left(\left\{(r, \varrho) \in \mathbb{R}^{2}: 0 \leq \varrho \leq r\right\}\right)$ so that $\varphi_{r}(\varrho)=h(r, \varrho) \sqrt{r-\varrho}$. This implies

$$
\begin{aligned}
g_{r}\left(\frac{\varrho}{r}\right) & =\frac{\varrho}{r} \dot{\varphi}_{r}(\varrho)+\partial_{r} \varphi_{r}(\varrho) \\
& =\frac{\varrho}{r}\left(\partial_{2} h(r, \varrho) \sqrt{r-\varrho}-\frac{h(r, \varrho)}{2 \sqrt{r-\varrho}}\right)+\left(\partial_{1} h(r, \varrho) \sqrt{r-\varrho}+\frac{h(r, \varrho)}{2 \sqrt{r-\varrho}}\right) \\
& =\left(2 \varrho \partial_{2} h(r, \varrho)+2 r \partial_{1} h(r, \varrho)+h(r, \varrho)\right) \frac{\sqrt{r-\varrho}}{2 r},
\end{aligned}
$$

hence $g_{r}$ is differentiable, and $g_{r}(1)=0$. Since the factors in equation (2.5) are continuous and $\varphi_{r}$ does not vanish on any interval, dividing (2.5) by $\sin ^{2 n-5} \varphi_{r}(y r)$ yields the equivalent equation

$$
\nu_{r}(y) g_{r}(y)+\lambda_{r}(y) \dot{g}_{r}(y) \equiv 0 .
$$

Period.Math. Hungar., 67:2 (2013), 187-209.

(c) Á. Kurusa 
If $\lambda_{r}(y) \neq 0$, then $\lambda_{r}$ does not vanish on an open interval $\mathcal{I} \ni y$, and on that interval one can multiply $(2.6)$ by

$$
\kappa_{r}(y):=\exp \left(\int \frac{\nu_{r}(y)-\dot{\lambda}_{r}(y)}{\lambda_{r}(y)} \mathrm{d} y\right)
$$

to arrive at the equivalent differential equation

$$
\begin{aligned}
\partial_{y}\left(\lambda_{r}(y) \kappa_{r}(y) g_{r}(y)\right) & =\partial_{y}\left(\lambda_{r}(y) \kappa_{r}(y)\right) g_{r}(y)+\lambda_{r}(y) \kappa_{r}(y) \dot{g}_{r}(y) \\
& =\nu_{r}(y) \kappa_{r}(y) g_{r}(y)+\lambda_{r}(y) \kappa_{r}(y) \dot{g}_{r}(y) \equiv 0 .
\end{aligned}
$$

This means that $\lambda_{r}(y) \kappa_{r}(y) g_{r}(y)$ does not depend on $y$ in the interval $\mathcal{I}$, therefore if $\lambda_{r}$ vanishes on any endpoint of $\mathcal{I}$, then $\lambda_{r}(y) \kappa_{r}(y) g_{r}(y) \equiv 0$ on the whole $\mathcal{I}$.

Thus, if $\lambda_{r}$ has a root in $[0,1]$, then $\lambda_{r} \kappa_{r} g_{r}$ vanishes on the whole $[0,1]$. If on the contrary $\lambda_{r}$ has no root in $[0,1]$, then $g_{r}(1)=0$ implies the vanishing of $\lambda_{r} \kappa_{r} g_{r}$ on the whole $[0,1]$.

As $\lambda_{r} \kappa_{r} g_{r}$ vanishes on the whole interval $[0,1]$, if $\lambda_{r}(y) \neq 0$ then $\kappa_{r}(y)>0$ implies $g_{r}(y)=0$. If on the contrary $\lambda_{r}(y)=0$, then equation (2.6) implies $g_{r}(y)=0$, because $\nu_{r}(y)>0$.

We conclude $g_{r} \equiv 0$ that means $\partial_{r}\left(\varphi_{r}(y r)\right) \equiv 0$. The proof is complete.

In the following theorem both inner and outer nice flowers appear. It shows that the petals of the flower of the dual of a rotational Radon transform $\mathrm{R}_{\mathcal{S}, \mu}$ are the inversions of the petals of $\mathcal{S}$ in the unit sphere, which is a generalization of Cormack's observation in [3].

In the theorem and its proof we identify the functions $f, g: \mathbb{R}^{n} \rightarrow \mathbb{R}$ by their counterparts $f \circ M$ and $g \circ M$ on $\mathbb{S}^{n-1} \times \mathbb{R}_{+}$, respectively, by the map $M: \mathbb{S}^{n-1} \times$ $\mathbb{R}_{+} \rightarrow \mathbb{R}^{n}$ with $(\omega, r) \mapsto r \omega$.

Theorem 2.2. Let $\mathcal{S}$ be a conformal flower defined by the curves $c_{r}^{\circ}(\varrho)=\varrho \omega_{\varphi_{r}^{\circ}(\varrho)}$, where $(\circ \in \pm)$, and define the flower $\mathcal{S}^{*}$ by the curves $c_{\varrho}^{\circ *}(r)=r \omega_{\psi_{\varrho}(r)}$, where $\psi_{\varrho}^{\circ}(r)=-\varphi_{r}^{\circ}(\varrho)$. Let $\mu$ be a rotational weight on the flower $\mathcal{S}$ and define the weight $\mu^{*}$ on $\mathcal{S}^{*}$ by $\varrho \mu_{\omega, \varrho}^{*}(r \bar{\omega})=r \mu_{\bar{\omega}, r}(\varrho \omega)$. Then the Radon transform $\mathrm{R}_{\mathcal{S}^{*}, \mu^{*}}$ is the dual $\mathrm{R}_{\mathcal{S}, \mu}^{*}$ of the Radon transform $\mathrm{R}_{\mathcal{S}, \mu}$.

Proof. Since the flower $\mathcal{S}$ is dilational, we observe that

$$
r \dot{\psi}_{\varrho}(r)=r \frac{\partial}{\partial r}\left(-\varphi_{1}\left(\frac{\varrho}{r}\right)\right)=\dot{\varphi}_{1}\left(\frac{\varrho}{r}\right) \frac{\varrho}{r}=\varrho \frac{\partial}{\partial \varrho}\left(\varphi_{1}\left(\frac{\varrho}{r}\right)\right)=\varrho \dot{\varphi}_{r}(\varrho) .
$$

We are proving that $\left\langle\mathrm{R}_{\mathcal{S}, \mu} f, g\right\rangle=\left\langle f, \mathrm{R}_{\mathcal{S}^{*}, \mu^{*}} g\right\rangle$. 
For dimension $n=2$, by equation (2.2) we have

$$
\begin{aligned}
&\left\langle\mathrm{R}_{\mathcal{S}, \mu} f, g\right\rangle= \int_{0}^{\infty} \int_{\mathbb{S}^{1}} \mathrm{R}_{\mathcal{S}, \mu} f(\bar{\omega}, r) g(r \bar{\omega}) r \mathrm{~d} \bar{\omega} \mathrm{d} r \\
&=\int_{0}^{\infty} \int_{-\pi}^{\pi} \int_{0}^{r} \sum_{\circ \in \pm} f\left(\varrho \omega_{\alpha+\varphi_{r}^{\circ}(\varrho)}\right) \mu_{\omega_{\alpha}, r}\left(\varrho \omega_{\alpha+\varphi_{r}^{\circ}(\varrho)}\right) \times \\
& \times \sqrt{1+\varrho^{2}\left(\dot{\varphi}_{r}^{\circ}(\varrho)\right)^{2}} \mathrm{~d} \varrho g\left(r \omega_{\alpha}\right) r \mathrm{~d} \alpha \mathrm{d} r .
\end{aligned}
$$

Using (2.7) this implies

$$
\begin{aligned}
\left\langle\mathrm{R}_{\mathcal{S}, \mu} f, g\right\rangle= & \int_{0}^{\infty} \int_{\varrho}^{\infty} \int_{-\pi}^{\pi} \sum_{\circ \in \pm} f\left(\varrho \omega_{\alpha+\varphi_{r}^{\circ}(\varrho)}\right) g\left(r \omega_{\alpha}\right) \mu_{\omega_{\alpha+\varphi_{r}^{\circ}(\varrho)}, \varrho}\left(r \omega_{\alpha}\right) \mathrm{d} \alpha \times \\
& \times \sqrt{1+\varrho^{2}\left(\dot{\varphi}_{r}^{\circ}(\varrho)\right)^{2}} \mathrm{~d} r \varrho \mathrm{d} \varrho \\
= & \int_{0}^{\infty} \int_{-\pi}^{\pi} f\left(\varrho \omega_{\beta}\right) \int_{\varrho}^{\infty} \sum_{\circ \in \pm} g\left(r \omega_{\beta+\psi_{\varrho}^{\circ}(r)}\right) \mu_{\omega_{\beta}, \varrho}^{*}\left(r \omega_{\beta+\psi_{\varrho}^{\circ}(r)}\right) \times \\
\times & \sqrt{1+r^{2}\left(\dot{\psi}_{\varrho}^{\circ}(r)\right)^{2}} \mathrm{~d} r \mathrm{~d} \beta \varrho \mathrm{d} \varrho \\
= & \int_{0}^{\infty} \int_{-\pi}^{\pi} f\left(\varrho \omega_{\beta}\right) \mathrm{R}_{\mathcal{S}^{*}, \mu^{*}} g\left(\omega_{\beta}, \varrho\right) \mathrm{d} \beta \varrho \mathrm{d} \varrho=\left\langle f, \mathrm{R}_{\mathcal{S}^{*}, \mu^{*}} g\right\rangle,
\end{aligned}
$$

which means $\mathrm{R}_{\mathcal{S}, \mu}^{*}=\mathrm{R}_{\mathcal{S}^{*}, \mu^{*}}$ as was stated.

For higher dimensions $n \geq 3$, we use equation (2.3) to get

$$
\begin{aligned}
\left\langle\mathrm{R}_{\mathcal{S}, \mu} f, g\right\rangle= & \int_{0}^{\infty} \int_{\mathbb{S}^{n-1}} \mathrm{R}_{\mathcal{S}, \mu} f(\bar{\omega}, r) g(r \bar{\omega}) r^{n-1} \mathrm{~d} \bar{\omega} \mathrm{d} r \\
= & \int_{0}^{\infty} \int_{\mathbb{S}^{n-1}} \int_{0}^{r} \int_{\mathbb{S}_{\bar{\omega}}^{n-2}} f\left(\varrho\left(\omega \sin \varphi_{r}(\varrho)+\bar{\omega} \cos \varphi_{r}(\varrho)\right)\right) \times \\
& \left.\times \mu_{\bar{\omega}, r}\left(\varrho \hat{\omega} \sin \varphi_{r}(\varrho)+\bar{\omega} \cos \varphi_{r}(\varrho)\right)\right) \mathrm{d} \omega \times \\
& \times \varrho^{n-2} \sin ^{n-2} \varphi_{r}(\varrho) \sqrt{1+\varrho^{2} \dot{\varphi}_{r}^{2}(\varrho)} \mathrm{d} \varrho g(r \bar{\omega}) r^{n-1} \mathrm{~d} \bar{\omega} \mathrm{d} r \\
= & \int_{0}^{\infty} \int_{\varrho}^{\infty} \int_{\mathbb{S}^{n-1}} \int_{\mathbb{S}^{n-1}} f(\varrho \hat{\omega}) g(r \bar{\omega}) \delta_{\cos \varphi_{r}(\varrho)}(\langle\hat{\omega}, \bar{\omega}\rangle) \mu_{\bar{\omega}, r}(\varrho \hat{\omega}) \mathrm{d} \hat{\omega} \mathrm{d} \bar{\omega} \times \\
& \times \varrho^{n-2} r^{n-1} \sin ^{n-2} \varphi_{r}(\varrho) \sqrt{1+\varrho^{2} \dot{\varphi}_{r}^{2}(\varrho)} \mathrm{d} r \mathrm{~d} \varrho .
\end{aligned}
$$

Period.Math. Hungar., $\mathbf{6 7 : 2}$ (2013), 187-209.

(c) Á. Kurusa 
Using (2.7) this results in

$$
\begin{aligned}
\left\langle\mathrm{R}_{\mathcal{S}, \mu} f, g\right\rangle= & \int_{0}^{\infty} \int_{\varrho}^{\infty} \int_{\mathbb{S}^{n-1}} \int_{\mathbb{S}^{n-1}} f(\varrho \hat{\omega}) g(r \bar{\omega}) \delta_{\cos \varphi_{r}(\varrho)}(\langle\hat{\omega}, \bar{\omega}\rangle) \mu_{\hat{\omega}, \varrho}^{*}(r \bar{\omega}) \mathrm{d} \bar{\omega} \mathrm{d} \hat{\omega} \times \\
& \times \varrho^{n-1} r^{n-2} \sin ^{n-2} \psi_{\varrho}(r) \sqrt{1+r^{2} \dot{\psi}_{\varrho}^{2}(r)} \mathrm{d} r \mathrm{~d} \varrho \\
= & \int_{\mathbb{S}^{n-1}} \int_{0}^{\infty} f(\varrho \hat{\omega}) \int_{\varrho}^{\infty} \int_{\mathbb{S}^{n-1}} g(r \bar{\omega}) \delta_{\cos \psi_{\varrho}(r)}(\langle\hat{\omega}, \bar{\omega}\rangle) \mu_{\hat{\omega}, \varrho}^{*}(r \bar{\omega}) \mathrm{d} \bar{\omega} \times \\
& \times r^{n-2} \sin ^{n-2} \psi_{\varrho}(r) \sqrt{1+r^{2} \dot{\psi}_{\varrho}^{2}(r)} \mathrm{d} r \varrho^{n-1} \mathrm{~d} \varrho \mathrm{d} \hat{\omega} \\
= & \int_{\mathbb{S}^{n-1}} \int_{0}^{\infty} f(\varrho \hat{\omega}) \mathrm{R}_{\mathcal{S}^{*}, \mu^{*}} g(\hat{\omega}, \varrho) \varrho^{n-1} \mathrm{~d} \varrho \mathrm{d} \hat{\omega}=\left\langle f, \mathrm{R}_{\mathcal{S}^{*}, \mu^{*}} g\right\rangle
\end{aligned}
$$

that shows $\mathrm{R}_{\mathcal{S}, \mu}^{*}=\mathrm{R}_{\mathcal{S}^{*}, \mu^{*}}$.

\section{Identifying properties of rotational Radon transforms}

A Radon transform $\mathrm{R}_{\mathcal{S}, \mu}$ is called rotationally invariant if $\mathrm{R}_{\mathcal{S}, \mu} f(\Phi \bar{\omega}, r)=$ $\mathrm{R}_{\mathcal{S}, \mu} f^{\Phi}(\bar{\omega}, r)$ for all $\Phi \in S O(n)$ and for all $f \in \mathrm{L}^{2}\left(\mathbb{R}^{n}\right)$, and it is called dilationally invariant if $\mathrm{R}_{\mathcal{S}, \mu} f(\bar{\omega}, p r)=p^{n-1} \mathrm{R}_{\mathcal{S}, \mu} f_{p}(\bar{\omega}, r)$ for all $p>0$ and for all $f \in \mathrm{L}^{2}\left(\mathbb{R}^{n}\right)$. We call a rotationally and dilationally invariant Radon transform $\mathrm{R}_{\mathcal{S}, \mu}$ conformally invariant.

Quinto proved in [13] that rotationally invariant generalized Radon transforms $\mathrm{R}_{\mathcal{H}, \mu}$ have rotational weight $\mu$. Here we generalize and sharpen Quinto's result using a different method and the attenuated hyper Pompeiu property which is defined in the Appendix of this article.

Theorem 3.1. Let $\mathcal{S}$ be a rotational flower, and the function $f \in \mathrm{L}^{2}\left(\mathbb{R}^{n}\right)$ be integrable on every petal of $\mathcal{S}$. Define the functions $f_{\varrho}(\omega):=f(\varrho \omega)$ on $\mathbb{S}^{n-1}$ for all $\varrho>0$ and the functions $g_{\ell}(\mathbf{x}):=|\mathbf{x}|^{\ell} f(\mathbf{x})$ for all $\ell \in \mathbb{N}$. Assume that

(1) if $n \geq 3$, then each $f_{\varrho}$ has the attenuated hyper Pompeiu property, and

(2) if $n=2$, then $\int_{\mathbb{S}^{1}} f_{\varrho}(\omega) \mathrm{d} \omega \neq 0$ and the equation $f_{\varrho}\left(\omega_{\alpha+\beta}\right)=f_{\varrho}\left(\omega_{\alpha}\right)$ has only finitely many solutions for $\alpha$ at any $\beta \in(-\pi, \pi]$.

If we have

(3) $\mathrm{R}_{\mathcal{S}, \mu} g_{\ell}(\Phi \bar{\omega}, r)=\mathrm{R}_{\mathcal{S}, \mu} g_{\ell}^{\Phi}(\bar{\omega}, r)$ for all $\Phi \in S O(n)$ and $\ell \in \mathbb{N}$, then $\mathrm{R}_{\mathcal{S}, \mu}$ is rotational.

Period.Math. Hungar., 67:2 (2013), 187-209.

(c) Á. Kurusa 
Proof. We have only to prove that the weight $\mu$ is rotational.

If the dimension $n$ is at least 3, then equation (2.3) and the conditions give for any $g_{\ell}$ that

$$
\begin{aligned}
& \mathrm{R}_{\mathcal{S}, \mu} g_{\ell}(\Phi \bar{\omega}, r) \\
&=\int_{0}^{r} \int_{\mathbb{S}_{\bar{\omega}}^{n-2}} g_{\ell}\left(\varrho \Phi\left(\omega \sin \varphi_{r}(\varrho)+\bar{\omega} \cos \varphi_{r}(\varrho)\right)\right) \times \\
& \times \mu_{\bar{\omega}, r}\left(\varrho\left(\omega \sin \varphi_{r}(\varrho)+\bar{\omega} \cos \varphi_{r}(\varrho)\right)\right) \times \\
& \times\left(\varrho \sin \varphi_{r}(\varrho)\right)^{n-2} \sqrt{1+\varrho^{2} \dot{\varphi}_{r}^{2}(\varrho)} \mathrm{d} \omega \mathrm{d} \varrho \\
&=\int_{0}^{r} \int_{\mathbb{S}^{n-1}} f_{\varrho}(\Phi \omega) \delta_{\cos \varphi_{r}(\varrho)}(\langle\omega, \bar{\omega}\rangle) \mu_{\bar{\omega}, r}(\varrho \omega) \mathrm{d} \omega \times \\
& \times\left(\varrho \sin \varphi_{r}(\varrho)\right)^{n-2} \sqrt{1+\varrho^{2} \dot{\varphi}_{r}^{2}(\varrho)} \varrho^{\ell} \mathrm{d} \varrho,
\end{aligned}
$$

where $\delta_{\cos \varphi_{r}(\varrho)}$ is the Dirac delta distribution supported at $\cos \varphi_{r}(\varrho)$ and $\langle.,$. denotes the usual inner product on $\mathbb{R}^{n}$.

By the Stone-Weierstrass Theorem the set of functions $\varrho^{\ell}(\ell \in \mathbb{N})$ is a complete base in $\mathrm{L}^{2}[0, r]$, whence equations $(3.1)(\ell \in \mathbb{N})$ determine the function

$$
\psi(\Phi \bar{\omega}, r, \varrho):=\int_{\mathbb{S}^{n-1}} f_{\varrho}(\Phi \omega) \delta_{\cos \varphi_{r}(\varrho)}(\langle\omega, \bar{\omega}\rangle) \mu_{\bar{\omega}, r}(\varrho \omega) \mathrm{d} \omega \quad(0<\varrho \leq r) .
$$

Substituting $\bar{\omega}=\Phi^{-1} \hat{\omega}$ and $\omega=\Phi^{-1} \tilde{\omega}$ results in

$$
\psi(\hat{\omega}, r, y)=\int_{\mathbb{S}_{\hat{\omega}}^{n-2}} f_{\varrho}(\omega) \mu_{\Phi^{-1} \hat{\omega}, r}\left(\varrho\left(\Phi^{-1} \omega \sin \varphi_{r}(\varrho)+\Phi^{-1} \hat{\omega} \cos \varphi_{r}(\varrho)\right)\right) \mathrm{d} \omega .
$$

Considering $\Phi$ in $\mathcal{K}_{\bar{\omega}}^{n}:=\{\Phi \in S O(n): \Phi \hat{\omega}=\hat{\omega}\} \cong S O(n-1)$, we arrive at

$$
\psi(\hat{\omega}, r, y)=\int_{\mathbb{S}_{\hat{\omega}}^{n-2}} f_{\varrho}(\omega) \mu_{\hat{\omega}, r}\left(\varrho\left(\Phi \omega \sin \varphi_{r}(\varrho)+\hat{\omega} \cos \varphi_{r}(\varrho)\right)\right) \mathrm{d} \omega
$$

for all $\Phi \in \mathcal{K}_{\bar{\omega}}^{n}$. Since $f_{\varrho}$ has the attenuated hyper Pompeiu property, its restriction on any great sphere in an everywhere dense set of great spheres $\mathbb{S}_{\hat{\omega}}^{n-2}$ has the Pompeiu property, which means that $\mu_{\hat{\omega}, r}\left(\varrho\left(\Phi \omega \sin \varphi_{r}(\varrho)+\hat{\omega} \cos \varphi_{r}(\varrho)\right)\right)$ is independent from $\Phi \in \mathcal{K}_{\hat{\omega}}^{n}$ on an everywhere dense set of $\hat{\omega} \in \mathbb{S}^{n-1}$. Since $\mu$ is continuous, this implies that the function $\mu_{\hat{\omega}, r}\left(\varrho\left(\omega \sin \varphi_{r}(\varrho)+\hat{\omega} \cos \varphi_{r}(\varrho)\right)\right)$ is independent from $\omega$ for every $\hat{\omega} \in \mathbb{S}^{n-1}$. By this and equation (3.2) we conclude

$$
\psi(\hat{\omega}, r, y)=\mu_{\Phi^{-1} \hat{\omega}, r}\left(\varrho \Phi^{-1}\left(\hat{\omega}^{\perp} \sin \varphi_{r}(\varrho)+\hat{\omega} \cos \varphi_{r}(\varrho)\right)\right) \int_{\mathbb{S}_{\hat{\omega}}^{n-2}} f_{\varrho}(\omega) \mathrm{d} \omega
$$

Period.Math. Hungar., $\mathbf{6 7 : 2}$ (2013), 187-209.

(c) Á. Kurusa 
for any unit vector $\hat{\omega}^{\perp}$ that is perpendicular to $\hat{\omega}$. This shows that $\mu_{\Phi \hat{\omega}, r}(\varrho \Phi \omega)$ is independent from $\Phi$ that is what was to be proved for higher dimensions $n \geq 3$.

Now, we proceed to the case of dimension $n=2$.

Equation (2.2) and the conditions imply for any $g_{\ell}$ that

$$
\begin{aligned}
\mathrm{R}_{\mathcal{S}, \mu} g_{\ell} & \left(\omega_{\alpha+\beta}, r\right) \\
= & \mathrm{R}_{\mathcal{S}, \mu} g_{\ell}^{\Phi}\left(\omega_{\alpha}, r\right) \\
= & \int_{0}^{r} \sum_{\circ \in \pm} g_{\ell}\left(\varrho \omega_{\alpha+\beta+\varphi_{r}^{\circ}(\varrho)}\right) \mu_{\omega_{\alpha}, r}\left(\varrho \omega_{\alpha+\varphi_{r}^{\circ}(\varrho)}\right) \sqrt{1+\varrho^{2}\left(\dot{\varphi}_{r}^{\circ}(\varrho)\right)^{2}} \mathrm{~d} \varrho \\
= & \int_{0}^{r} \sum_{\circ \in \pm} f_{\varrho}\left(\omega_{\alpha+\beta+\varphi_{r}^{\circ}(\varrho)}\right) \mu_{\omega_{\alpha}, r}\left(\varrho \omega_{\alpha+\varphi_{r}^{\circ}(\varrho)}\right) \sqrt{1+\varrho^{2}\left(\dot{\varphi}_{r}^{\circ}(\varrho)\right)^{2}} \varrho^{\ell} \mathrm{d} \varrho,
\end{aligned}
$$

where $\Phi \in S O(2)$ is the anticlockwise rotation by angle $\beta$. By the StoneWeierstrass Theorem the set of functions $\varrho^{\ell}(\ell \in \mathbb{N})$ is a complete base in $\mathrm{L}^{2}[0, r]$, whence equations $(3.4)(\ell \in \mathbb{N})$ determine the function

$$
\psi(\alpha+\beta, r, \varrho):=\sum_{\circ \in \pm} f_{\varrho}\left(\omega_{\alpha+\beta+\varphi_{r}^{\circ}(\varrho)}\right) \mu_{\omega_{\alpha}, r}\left(\varrho \omega_{\alpha+\varphi_{r}^{\circ}(\varrho)}\right) \sqrt{1+\varrho^{2}\left(\dot{\varphi}_{r}^{\circ}(\varrho)\right)^{2}}
$$

for $\varrho \in(0, r]$. Integrating this by $\beta$ on $(-\pi, \pi]$ we get

$$
\bar{\psi}(r, \varrho):=\frac{\int_{\mathbb{S}^{1}} \psi(\alpha+\beta, r, \varrho) \mathrm{d} \beta}{\int_{\mathbb{S}^{1}} f_{\varrho}\left(\omega_{\alpha+\beta+\varphi_{r}^{\circ}(\varrho)}\right) \mathrm{d} \beta}=\sum_{\circ \in \pm} \mu_{\omega_{\alpha}, r}\left(\varrho \omega_{\alpha+\varphi_{r}^{\circ}(\varrho)}\right) \sqrt{1+\varrho^{2}\left(\dot{\varphi}_{r}^{\circ}(\varrho)\right)^{2}}
$$

Using this, equation (3.5) gives

$$
\begin{aligned}
& \psi(\alpha+\beta, r, \varrho) \\
& =\bar{\psi}(r, \varrho) f_{\varrho}\left(\omega_{\alpha+\beta+\varphi_{r}^{-}(\varrho)}\right)+ \\
& \quad+\left(f_{\varrho}\left(\omega_{\alpha+\beta+\varphi_{r}^{+}(\varrho)}\right)-f_{\varrho}\left(\omega_{\alpha+\beta+\varphi_{r}^{-}(\varrho)}\right)\right) \mu_{\omega_{\alpha}, r}\left(\varrho \omega_{\alpha+\varphi_{r}^{+}(\varrho)}\right) \sqrt{1+\varrho^{2}\left(\dot{\varphi}_{r}^{+}(\varrho)\right)^{2}}
\end{aligned}
$$

which by (2) and the continuity of $\mu$ implies

$$
\frac{\psi(\alpha+\beta, r, \varrho)-\bar{\psi}(r, \varrho) f_{\varrho}\left(\omega_{\alpha+\beta+\varphi_{r}^{-}(\varrho)}\right)}{f_{\varrho}\left(\omega_{\alpha+\beta+\varphi_{r}^{+}(\varrho)}\right)-f_{\varrho}\left(\omega_{\alpha+\beta+\varphi_{r}^{-}(\varrho)}\right)}=\mu_{\omega_{\alpha}, r}\left(\varrho \omega_{\alpha+\varphi_{r}^{+}(\varrho)}\right) \sqrt{1+\varrho^{2}\left(\dot{\varphi}_{r}^{+}(\varrho)\right)^{2}} .
$$

The right-hand side shows that the left-hand side does not depend on $\beta$, whence the value $\beta=-\alpha$ implies that the right-hand side does not depend on $\alpha$. This implies via (3.6) that $\mu_{\omega_{\alpha}, r}\left(\varrho \omega_{\alpha+\varphi_{r}^{-}(\varrho)}\right)$ is also independent from $\alpha$.

Period.Math. Hungar., 67:2 (2013), 187-209.

(c) Á. Kurusa 
The theorem is proved.

Our result implies immediately that each rotationally invariant Radon transform with rotational flower is rotational, which is a generalization and sharpening of Quinto's result in [13, Proposition 2.2].

The following result clarifies why Quinto's [13, Proposition 2.2] differs from our Theorem 3.1 in the plane. The difference occurs because Quinto uses $O(2)$ while we used $S O(2)$ in the definition of rotational invariance, and because $\mathcal{H}$ is not only rotational but also symmetric.

Theorem 3.2. Let $\mathrm{R}_{\mathcal{S}, \mu}$ be a rotational Radon transform in the plane, and let the function $f \in \mathrm{L}^{2}\left(\mathbb{R}^{2}\right)$ be integrable on every petal of $\mathcal{S}$. Define the functions $f_{\varrho}(\omega):=f(\varrho \omega)$ on $\mathbb{S}^{1}$ for all $\varrho>0$ and the functions $g_{\ell}(\mathbf{x}):=|\mathbf{x}|^{\ell} f(\mathbf{x})$ for all $\ell \in \mathbb{N}$. Assume that

(1) the equation $f_{\varrho}\left(\omega_{\alpha+\beta}\right)=f_{\varrho}\left(\omega_{\alpha}\right)$ has only finitely many solutions for $\alpha$ at any $\beta \in(-\pi, \pi]$.

If $\mathcal{S}$ is symmetric and

(2) $\mathrm{R}_{\mathcal{S}, \mu} g_{\ell}(\Gamma \bar{\omega}, r)=\mathrm{R}_{\mathcal{S}, \mu} g_{\ell}^{\Gamma}(\bar{\omega}, r)$ for all $\ell \in \mathbb{N}$, where $\Gamma$ is the reflection to the $x$-axis,

then $\mathrm{R}_{\mathcal{S}, \mu}$ is symmetric.

Proof. We only need to prove that $\mu$ is symmetric.

As the flower is symmetric, there is a function $\varphi_{r}(\varrho)$ so that $\varphi_{r}^{ \pm}(\varrho)= \pm \varphi_{r}(\varrho)$. Now equation (3.4) at $\beta=0$ reads

$$
\begin{aligned}
& \mathrm{R}_{\mathcal{S}, \mu} g_{\ell}\left(\omega_{\alpha}, r\right) \\
& \quad=\int_{0}^{r} \sum_{i \in\{-1,1\}} f_{\varrho}\left(\omega_{\alpha+i \varphi_{r}(\varrho)}\right) \mu_{\omega_{0}, r}\left(\varrho \omega_{i \varphi_{r}(\varrho)}\right) \sqrt{1+\varrho^{2} \dot{\varphi}_{r}^{2}(\varrho)} \varrho^{\ell} \mathrm{d} \varrho,
\end{aligned}
$$

because $\mu$ is rotational. Using condition (2), we get in the same way that

$$
\begin{aligned}
\mathrm{R}_{\mathcal{S}, \mu} g_{\ell} & \left(\omega_{\alpha}, r\right) \\
& =\mathrm{R}_{\mathcal{S}, \mu} g_{\ell}^{\Gamma}\left(\omega_{-\alpha}, r\right) \\
& =\int_{0}^{r} \sum_{i \in\{-1,1\}} f_{\varrho}\left(\omega_{\alpha-i \varphi_{r}(\varrho)}\right) \mu_{\omega_{0}, r}\left(\varrho \omega_{i \varphi_{r}(\varrho)}\right) \sqrt{1+\varrho^{2} \dot{\varphi}_{r}^{2}(\varrho)} \varrho^{\ell} \mathrm{d} \varrho .
\end{aligned}
$$

By the Stone-Weierstrass Theorem the set of functions $\varrho^{\ell}(\ell \in \mathbb{N})$ is a complete base in $\mathrm{L}^{2}[0, r]$, whence the difference of equations (3.7) and (3.8) implies

$$
0=\sum_{i \in\{-1,1\}}\left(f_{\varrho}\left(\omega_{\alpha+i \varphi_{r}(\varrho)}\right)-f_{\varrho}\left(\omega_{\alpha-i \varphi_{r}(\varrho)}\right)\right) \mu_{\omega_{0}, r}\left(\varrho \omega_{i \varphi_{r}(\varrho)}\right)
$$

Period.Math. Hungar., 67:2 (2013), 187-209.

(c) Á. Kurusa 
for all $\varrho \in(0, r]$. By condition (1) the coefficients $f_{\varrho}\left(\omega_{\alpha+i \varphi_{r}(\varrho)}\right)-f_{\varrho}\left(\omega_{\alpha-i \varphi_{r}(\varrho)}\right)$ in (3.23) may only vanish for finitely many $\alpha$, therefore except for finitely many $\alpha$ we have $\mu_{\omega_{\alpha}, r}\left(\varrho \omega_{\alpha+\varphi_{r}(\varrho)}\right)=\mu_{\omega_{\alpha}, r}\left(\varrho \omega_{\alpha-\varphi_{r}(\varrho)}\right)$. As $\mu$ is continuous, this proves the theorem.

Theorem 3.3. Let $\mathrm{R}_{\mathcal{S}, \mu}$ be a symmetric rotational Radon transform, and let $f \in$ $\mathrm{L}_{*}^{2}\left(\mathbb{R}^{n}\right)$ be a radial function of compact support without vanishing moments so that $\mathrm{R}_{\mathcal{S}, \mu} f(\bar{\omega}, p r)=p^{n-1} \mathrm{R}_{\mathcal{S}, \mu} f_{p}(\bar{\omega}, r)$ for all $p>0$. If either $\mathcal{S}$ or $\mu$ is dilational, then $\mathrm{R}_{\mathcal{S}, \mu}$ is conformal.

Proof. Since $\mu$ is a rotational weight, we have a real function $\bar{\mu}_{r}$ so that $(2.1)$ is fulfilled. Since $f$ is radial, there is a real function $\hat{f}$ that satisfies $f(\mathbf{x})=\hat{f}(|\mathbf{x}|)$ for all $\mathbf{x} \in \mathbb{R}^{n}$.

As $\mathcal{S}$ is rotational and symmetric, equations (2.3) and (2.2) give

$$
\begin{aligned}
\mathrm{R}_{\mathcal{S}, \mu} & f(\bar{\omega}, p r) \\
& =p^{n-1} \mathrm{R}_{\mathcal{S}, \mu} f_{p}(\bar{\omega}, r) \\
& =\left|\mathbb{S}^{n-2}\right| p^{n-1} \int_{0}^{r} \hat{f}(p \varrho) \bar{\mu}_{r}(\varrho)\left(\varrho \sin \varphi_{r}(\varrho)\right)^{n-2} \sqrt{1+\varrho^{2} \dot{\varphi}_{r}^{2}(\varrho)} \mathrm{d} \varrho
\end{aligned}
$$

for all $p, r \in[0, \infty)$, where we set $\left|\mathbb{S}^{0}\right|=2$.

Integrating $(3.10)$ over $[0, \infty)$ with respect to $q^{-k} \mathrm{~d} q(2 \leq k \in \mathbb{N})$, where $q=r p$, then substituting $t=p \varrho$ and finally substituting $\varrho=y r$ we get

$$
\begin{aligned}
& \frac{1}{\left|\mathbb{S}^{n-2}\right|} \int_{0}^{\infty} \mathrm{R}_{\mathcal{S}, \mu} f(\bar{\omega}, q) q^{-k} \mathrm{~d} q \\
& \quad=\int_{0}^{\infty} \hat{f}(t) t^{n-1-k} \mathrm{~d} t \int_{0}^{1} \bar{\mu}_{r}(y r) y^{k-2} \sin ^{n-2} \varphi_{r}(y r) \sqrt{1+(y r)^{2} \dot{\varphi}_{r}^{2}(y r)} \mathrm{d} y .
\end{aligned}
$$

Dividing the equation by $\int_{0}^{\infty} f(t) t^{n-1-k} \mathrm{~d} t$ results in

$$
c_{k}=\int_{0}^{1} y^{k-2} \bar{\mu}_{r}(y r) \sin ^{n-2} \varphi_{r}(y r) \sqrt{1+y^{2} r^{2} \dot{\varphi}_{r}^{2}(y r)} \mathrm{d} y, \quad 2 \leq k \in \mathbb{N},
$$

where $c_{k}$ is a real number for each $k$.

By the Stone-Weierstrass Theorem the set of functions $y^{k}(k \in \mathbb{N})$ is a complete base in $\mathrm{L}^{2}[0,1]$, whence the constants $c_{k}(k \in \mathbb{N})$ determine the function $\psi(y):=\bar{\mu}_{r}(y r) \sin ^{n-2} \varphi_{r}(y r) \sqrt{1+y^{2} r^{2} \dot{\varphi}_{r}^{2}(y r)}(0<y \leq 1)$ which is therefore independent from $r$.

Period.Math. Hungar., $\mathbf{6 7 : 2}$ (2013), 187-209.

(c) Á. Kurusa 
Now, if $\mathcal{S}$ is dilational, then $\sin ^{n-2} \varphi_{r}(y r) \sqrt{1+y^{2} r^{2} \dot{\varphi}_{r}^{2}(y r)}$ depends only on $y$, therefore $\bar{\mu}_{r}(y r)$ is independent from $r$, that proves the conformality of $\mu$. If $\mu$ is dilational, then $\bar{\mu}_{r}(y r)$ depends only on $y$, whence $\sin ^{n-2} \varphi_{r}(y r) \sqrt{1+y^{2} r^{2} \dot{\varphi}_{r}^{2}(y r)}$ is independent from $r$, which by Lemma 2.1 implies the conformality of $\mathcal{S}$.

The theorem is proved.

The previous two theorems imply that a conformally invariant Radon transform with conformal flower is conformal, but as radial functions are not suitable for Theorem 3.1, to check conformality we need different test functions for Theorems 3.1 and 3.3. In the following theorem we use only one function to get the conformality of a Radon transform.

Theorem 3.4. Let $\mathcal{S}$ be a conformal flower and let $f \in \mathrm{L}_{*}^{2}\left(\mathbb{R}^{n}\right)$ be of compact support such that

(1) the coefficients $f_{l, m}$ in the spherical harmonic expansion of $f$ are independent in $\mathrm{L}^{2}(\mathbb{R})$,

(2) for each $\ell \in \mathbb{N}$ the function $g(\mathbf{x}):=|\mathbf{x}|^{\ell} f(\mathbf{x})$ satisfies

(2a) $\mathrm{R}_{\mathcal{S}, \mu} g(\Phi \bar{\omega}, r)=\mathrm{R}_{\mathcal{S}, \mu} g^{\Phi}(\bar{\omega}, r)$ for all $\Phi \in S O(n)$, and

(2b) $\mathrm{R}_{\mathcal{S}, \mu} g(\bar{\omega}, p r)=p^{n-1} \mathrm{R}_{\mathcal{S}, \mu} g_{p}(\bar{\omega}, r)$ for all $p>0$.

Then $\mathrm{R}_{\mathcal{S}, \mu}$ is conformal.

Proof. Before entrenching ourself into the proof let us consider some corollaries of condition (1). First of all, there is an $0<R \in \mathbb{R}$ so that $f \in \mathrm{L}_{*}^{2}\left(R \mathbb{B}^{n}\right)$, where $\mathbb{B}^{n}$ is the unit ball of $\mathbb{R}^{n}$, therefore the coefficients $f_{l, m}$ in the spherical harmonic expansion $f(q \omega)=\sum_{l, m}^{\infty} f_{l, m}(q) Y_{l, m}(\omega)$ are all in $\mathrm{L}^{2}[0, R]$. These coefficients are independent in $\mathrm{L}^{2}[0, R]$, therefore the infinite-dimensional analogue of the Gram-Schmidt process gives an orthogonal system of compactly supported functions $f_{l, m}^{*} \in \mathrm{L}^{2}[0, R]$ so that

$$
\int_{0}^{\infty} f_{l^{\prime}, m^{\prime}}^{*}(q) f_{l, m}(q) \mathrm{d} q= \begin{cases}1, & \text { if }\left(l^{\prime}, m^{\prime}\right)=(l, m) \\ 0, & \text { if }\left(l^{\prime}, m^{\prime}\right) \neq(l, m) .\end{cases}
$$

Then, by the Stone-Weierstrass Theorem there is a sequence of polynomials $\tilde{p}_{l^{\prime}, m^{\prime}}^{j}$ $(j \in \mathbb{N})$ tending uniformly to $f_{l^{\prime}, m^{\prime}}^{*}$ on $[0, R]$, and therefore

$$
F_{\left\langle\tilde{p}_{l^{\prime}, m^{\prime}}^{j}\right\rangle}(\omega)=\sum_{l, m}^{\infty}\left(\int_{0}^{\infty} \tilde{p}_{l^{\prime}, m^{\prime}}^{j}(q) f_{l, m}(q) \mathrm{d} q Y_{l, m}(\omega)\right) \rightarrow Y_{l^{\prime}, m^{\prime}}(\omega) .
$$

where $F_{\langle\hat{p}\rangle}(\omega):=\int_{0}^{\infty} \hat{p}(q) f(q \omega) \mathrm{d} q$ for all polynomials $\hat{p}$. 
Having this result we start proving our theorem in dimensions higher than 2 . Then, by (2.3) and the conditions we have for any $g$ that

$$
\begin{aligned}
& \mathrm{R}_{\mathcal{S}, \mu} g(\Phi \bar{\omega}, p r) \\
& =p^{n-1} \mathrm{R}_{\mathcal{S}, \mu} g_{p}^{\Phi}(\bar{\omega}, r) \\
& =p^{n-1} \int_{0}^{r} \int_{\mathbb{S}_{\bar{\omega}}^{n-2}} g(p \varrho \Phi(\omega \\
&
\end{aligned}
$$

that is

$$
\begin{aligned}
\mathrm{R}_{\mathcal{S}, \mu} g(\Phi \bar{\omega}, p r) \\
=p^{n-1} \int_{0}^{r} \int_{\mathbb{S}^{n-1}} g(p \varrho \Phi \omega) \delta_{\cos \varphi_{r}(\varrho)}(\langle\omega, \bar{\omega}\rangle) \mu_{\bar{\omega}, r}(\varrho \omega) \mathrm{d} \omega \times \\
\times\left(\varrho \sin \varphi_{r}(\varrho)\right)^{n-2} \sqrt{1+\varrho^{2} \dot{\varphi}_{r}^{2}(\varrho)} \mathrm{d} \varrho \\
=(p r)^{n-1} \int_{0}^{1} \int_{\mathbb{S}^{n-1}} g(p y r \Phi \omega) \delta_{\cos \varphi_{1}(y)}(\langle\omega, \bar{\omega}\rangle) \mu_{\bar{\omega}, r}(y r \omega) \mathrm{d} \omega \times \\
\times y^{n-2} \sin ^{n-2} \varphi_{1}(y) \sqrt{1+y^{2} \dot{\varphi}_{1}^{2}(y)} \mathrm{d} y,
\end{aligned}
$$

where $\delta_{\cos \varphi_{r}(\varrho)}$ is the Dirac delta distribution offset to $\cos \varphi_{r}(\varrho)$ and $\langle.,$.$\rangle denotes$ the usual inner product on $\mathbb{R}^{n}$.

Integrating $\mathrm{R}_{\mathcal{S}, \mu} g(\Phi \bar{\omega}, t)$ by $t^{-k} \mathrm{~d} t(2 \leq k \in \mathbb{N})$ over $[0, \infty)$, where $t=p r$, then using (3.12) in which substituting $q=$ pyr leads to

$$
\begin{array}{r}
h_{k}(\Phi \bar{\omega}):=\int_{0}^{\infty} \mathrm{R}_{\mathcal{S}, \mu} g(\Phi \bar{\omega}, t) t^{-k} \mathrm{~d} t=r^{1-k} \int_{0}^{\infty} \mathrm{R}_{\mathcal{S}, \mu} g(\Phi \bar{\omega}, p r) p^{-k} \mathrm{~d} p \\
=\int_{0}^{1} \int_{\mathbb{S}^{n-1}} \int_{0}^{\infty} q^{n-1-k} g(q \Phi \omega) \mathrm{d} q \delta_{\cos \varphi_{1}(y)}(\langle\omega, \bar{\omega}\rangle) \mu_{\bar{\omega}, r}(y r \omega) \mathrm{d} \omega \times \\
\times y^{k-2} \sin ^{n-2} \varphi_{1}(y) \sqrt{1+y^{2} \dot{\varphi}_{1}^{2}(y)} \mathrm{d} y .
\end{array}
$$

Choosing $g(\mathbf{x}):=|\mathbf{x}|^{\ell+k-n+1} f(\mathbf{x})(\ell \in \mathbb{N})$ we get

$$
\begin{array}{r}
h_{k, \ell}(\Phi \bar{\omega}):=\int_{0}^{1} \int_{\mathbb{S}^{n-1}} \int_{0}^{\infty} q^{\ell} f(q \Phi \omega) \mathrm{d} q \delta_{\cos \varphi_{1}(y)}(\langle\omega, \bar{\omega}\rangle) \mu_{\bar{\omega}, r}(y r \omega) \mathrm{d} \omega \times \\
\times y^{k-2} \sin ^{n-2} \varphi_{1}(y) \sqrt{1+y^{2} \dot{\varphi}_{1}^{2}(y)} \mathrm{d} y .
\end{array}
$$

Period.Math. Hungar., 67:2 (2013), 187-209.

(c) Á. Kurusa 
Since this is valid for all integers $k \geq 2$, again the Stone-Weierstrass Theorem gives that

$$
\int_{\mathbb{S}^{n-1}} \int_{0}^{\infty} q^{\ell} f(q \Phi \omega) \mathrm{d} q \delta_{\cos \varphi_{1}(y)}(\langle\omega, \bar{\omega}\rangle) \mu_{\bar{\omega}, r}(y r \omega) \mathrm{d} \omega
$$

depends only on $y \in[0,1]$ and on $\Phi \bar{\omega}$, hence it is a function $h_{\ell}^{*}(\Phi \bar{\omega}, y)$ (this can be determined from the functions $h_{k, k}(\Phi \bar{\omega})$ by (3.13)). We thus have for any polynomial $\hat{p}$ on $\mathbb{R}$ the function

$$
h_{\langle\hat{p}\rangle}^{*}(\Phi \bar{\omega}, y)=\int_{\mathbb{S}^{n-1}} \int_{0}^{\infty} \hat{p}(q) f(q \Phi \omega) \mathrm{d} q \delta_{\cos \varphi_{1}(y)}(\langle\omega, \bar{\omega}\rangle) \mu_{\bar{\omega}, r}(y r \omega) \mathrm{d} \omega,
$$

where $(\bar{\omega}, y) \in \mathbb{S}^{n-1} \times[0,1]$. Substituting $\Phi=\Psi^{-1}, \bar{\omega}=\Psi \hat{\omega}, \omega=\Psi \tilde{\omega}$, then $\hat{\omega}=\bar{\omega}$ and finally $\tilde{\omega}=\omega$, equation (3.14) can be written as

$$
h_{\langle\hat{p}\rangle}^{*}(\bar{\omega}, y)=\int_{\mathbb{S}^{n-1}} F_{\langle\hat{p}\rangle}(\omega) \delta_{\cos \varphi_{1}(y)}(\langle\omega, \bar{\omega}\rangle) \mu_{\Psi \bar{\omega}, r}(r y \Psi \omega) \mathrm{d} \omega
$$

where $F_{\langle\hat{p}\rangle}(\omega)=\int_{0}^{\infty} \hat{p}(q) f(q \omega) \mathrm{d} q$. Taking into account the left-hand side's independence from $\Psi$ we get

$$
0=\int_{\mathbb{S}^{n-1}} F_{\langle\hat{p}\rangle}(\omega) \delta_{\cos \varphi_{1}(y)}(\langle\omega, \bar{\omega}\rangle)\left(\mu_{\bar{\omega}, r}(r y \Psi \omega)-\mu_{\bar{\omega}, r}(r y \omega)\right) \mathrm{d} \omega, \quad \Psi \in \mathcal{K}_{\bar{\omega}}^{n},
$$

where $\mathcal{K}_{\bar{\omega}}^{n}:=\{\Psi \in S O(n): \Psi \bar{\omega}=\bar{\omega}\} \cong S O(n-1)$. Substituting the sequence of polynomials $\tilde{p}_{l^{\prime}, m^{\prime}}^{j}(j \in \mathbb{N})$ leads to

$$
0=\int_{\mathbb{S}^{n-1}} Y_{l^{\prime}, m^{\prime}}(\omega) \delta_{\cos \varphi_{1}(y)}(\langle\omega, \bar{\omega}\rangle)\left(\mu_{\bar{\omega}, r}(r y \Phi \omega)-\mu_{\bar{\omega}, r}(r y \omega)\right) \mathrm{d} \omega
$$

by equation (3.11).

As the spherical harmonics $Y_{l^{\prime}, m^{\prime}}$ constitute a base in $\mathrm{L}^{2}\left(\mathbb{S}^{n-1}\right)$, this implies $\mu_{\bar{\omega}, r}(r y \Phi \omega)=\mu_{\bar{\omega}, r}(r y \omega)$ for all $\omega \in \mathbb{S}^{n-1}$ satisfying $\langle\omega, \bar{\omega}\rangle=\cos \varphi_{1}(y)$ and for all $\Phi \in \mathcal{K}_{\bar{\omega}}^{n}$. Taking this into account in considering equation (3.15) we get

$$
h_{\langle\hat{p}\rangle}^{*}(\bar{\omega}, y)=\mu_{\Psi \bar{\omega}, r}\left(r y \Psi \bar{\omega}^{\perp}\right) \int_{\mathbb{S}^{n-1}} F_{\langle\hat{p}\rangle}(\omega) \delta_{\cos \varphi_{1}(y)}(\langle\omega, \bar{\omega}\rangle) \mathrm{d} \omega
$$

where $\bar{\omega}^{\perp}$ is any unit vector perpendicular to $\bar{\omega}$. This implies that $\mu$ is conformal, that proves the statement in dimensions higher than 2 .

Period.Math. Hungar., 67:2 (2013), 187-209.

(c) Á. Kurusa 
Now we proceed to the case of the plane, i.e., dimension $n=2$. By $(2.2)$ and the conditions we have for any $g$ and $\beta \in(-\pi, \pi]$, where $\Phi$ is the anticlockwise rotation by $\beta$, that

$$
\begin{aligned}
& \mathrm{R}_{\mathcal{S}, \mu} g\left(\omega_{\alpha+\beta}, p r\right) \\
& \quad=p \mathrm{R}_{\mathcal{S}, \mu} g_{p}^{\Phi}\left(\omega_{\alpha}, r\right) \\
& \quad=p \int_{0}^{r} \sum_{\circ \in \pm} g\left(p \varrho \omega_{\alpha+\beta+\varphi_{r}^{\circ}(\varrho)}\right) \mu_{\omega_{\alpha}, r}\left(\varrho \omega_{\alpha+\varphi_{r}^{\circ}(\varrho)}\right) \sqrt{1+\varrho^{2}\left(\dot{\varphi}_{r}^{\circ}(\varrho)\right)^{2}} \mathrm{~d} \varrho \\
& \quad=p r \int_{0}^{1} \sum_{\circ \in \pm} g\left(p r y \omega_{\alpha+\beta+\varphi_{1}^{\circ}(y)}\right) \mu_{\omega_{\alpha}, r}\left(r y \omega_{\alpha+\varphi_{1}^{\circ}(y)}\right) \sqrt{1+y^{2}\left(\dot{\varphi}_{1}^{\circ}(y)\right)^{2}} \mathrm{~d} y .
\end{aligned}
$$

Integrating $\mathrm{R}_{\mathcal{S}, \mu} g\left(\omega_{\alpha+\beta}, t\right)$ by $t^{-k} \mathrm{~d} t(2 \leq k \in \mathbb{N})$ over $[0, \infty)$, where $t=p r$, then using (3.16) in which substituting $q=$ pyr leads to

$$
\begin{aligned}
h_{k}\left(\omega_{\alpha+\beta}\right):= & \int_{0}^{\infty} \mathrm{R}_{\mathcal{S}, \mu} g\left(\omega_{\alpha+\beta}, t\right) t^{-k} \mathrm{~d} t=r^{1-k} \int_{0}^{\infty} \mathrm{R}_{\mathcal{S}, \mu} g\left(\omega_{\alpha+\beta}, p r\right) p^{-k} \mathrm{~d} p \\
= & \int_{0}^{1} \sum_{\circ \in \pm} \int_{0}^{\infty} g\left(q \omega_{\alpha+\beta+\varphi_{1}^{\circ}(y)}\right) q^{1-k} \mathrm{~d} q \times \\
& \times \mu_{\omega_{\alpha}, r}\left(r y \omega_{\alpha+\varphi_{1}^{\circ}(y)}\right) y^{k-2} \sqrt{1+y^{2}\left(\dot{\varphi}_{1}^{\circ}(y)\right)^{2}} \mathrm{~d} y .
\end{aligned}
$$

Choosing $g(\mathbf{x}):=|\mathbf{x}|^{\ell+k-1} f(\mathbf{x})(\ell \in \mathbb{N})$ we get

$$
\begin{aligned}
h_{k, \ell}\left(\omega_{\alpha+\beta}\right):=\int_{0}^{1} \sum_{\circ \in \pm} \int_{0}^{\infty} & q^{\ell} f\left(q \omega_{\alpha+\beta+\varphi_{1}^{\circ}(y)}\right) \mathrm{d} q \times \\
& \times \mu_{\omega_{\alpha}, r}\left(r y \omega_{\alpha+\varphi_{1}^{\circ}(y)}\right) \sqrt{1+y^{2}\left(\dot{\varphi}_{1}^{\circ}(y)\right)^{2}} y^{k-2} \mathrm{~d} y .
\end{aligned}
$$

Since this is valid for all integers $k \geq 2$, again the Stone-Weierstrass Theorem gives that

$$
\sum_{\circ \in \pm} \int_{0}^{\infty} q^{\ell} f\left(q \omega_{\alpha+\beta+\varphi_{1}^{\circ}(y)}\right) \mathrm{d} q \mu_{\omega_{\alpha}, r}\left(r y \omega_{\alpha+\varphi_{1}^{\circ}(y)}\right) \sqrt{1+y^{2}\left(\dot{\varphi}_{1}^{\circ}(y)\right)^{2}}
$$

depends only on $y \in[0,1]$ and on $\omega_{\alpha+\beta}$, hence it is a function $h_{\ell}^{*}\left(\omega_{\alpha+\beta}, y\right)$ (this can be determined from the functions $h_{k, k}\left(\omega_{\alpha+\beta}\right)$ by $\left.(3.17)\right)$. We thus have for any polynomial $\hat{p}$ on $\mathbb{R}$ the function

$$
h_{\langle\hat{p}\rangle}^{*}\left(\omega_{\alpha+\beta}, y\right)=\sum_{\circ \in \pm} F_{\langle\hat{p}\rangle}\left(\omega_{\alpha+\beta+\varphi_{1}^{\circ}(y)}\right) \mu_{\omega_{\alpha}, r}\left(r y \omega_{\alpha+\varphi_{1}^{\circ}(y)}\right) \sqrt{1+y^{2}\left(\dot{\varphi}_{1}^{\circ}(y)\right)^{2}}
$$

Period.Math. Hungar., $\mathbf{6 7 : 2}$ (2013), 187-209.

(c) Á. Kurusa 
where $y \in[0,1]$. Substituting $\beta=-\gamma, \alpha=\hat{\alpha}+\gamma$, then $\hat{\alpha}=\alpha$ and finally $\gamma=\beta$, equation (3.18) can be written as

$$
h_{\langle\hat{p}\rangle}^{*}\left(\omega_{\alpha}, y\right)=\sum_{\circ \in \pm} F_{\langle\hat{p}\rangle}\left(\omega_{\alpha+\varphi_{1}^{\circ}(y)}\right) \mu_{\omega_{\alpha}+\beta, r}\left(r y \omega_{\alpha+\beta+\varphi_{1}^{\circ}(y)}\right) \sqrt{1+y^{2}\left(\dot{\varphi}_{1}^{\circ}(y)\right)^{2}}
$$

Fixing $\alpha$ and $y$, this is a linear equation of the unknowns $\mu_{\omega_{\alpha+\beta}, r}\left(r y \omega_{\alpha+\beta+\varphi_{1}^{\circ}(y)}\right)$ for each polynomial $\hat{p}$. The system of these linear equations can be degenerated for a pair $(\alpha, y)$ if and only if the vectors $\left(F_{\langle\hat{p}\rangle}\left(\omega_{\alpha+\gamma}\right), F_{\langle\hat{p}\rangle}\left(\omega_{\alpha}\right)\right)$ are real multiples of each other for all polynomials $\hat{p}$, where $\gamma=\varphi_{1}^{+}(y)-\varphi_{1}^{-}(y) \neq 0$.

As an indirect step, assume now that system (3.19) degenerates at some $\alpha$ and $\gamma \neq 0$, which means that $\left(F_{\langle\hat{p}\rangle}\left(\omega_{\alpha+\gamma}\right), F_{\langle\hat{p}\rangle}\left(\omega_{\alpha}\right)\right)$ are real multiples of each other for all polynomials $\hat{p}$. Choosing the sequences of polynomials $\hat{p}_{j}:=\tilde{p}_{l^{\prime}, m^{\prime}}^{j}(j \in \mathbb{N})$ and $\bar{p}_{k}:=\tilde{p}_{l, m}^{k}(k \in \mathbb{N})$, and taking $k \rightarrow \infty$, we obtain

$$
\begin{aligned}
\left(F_{\left\langle\hat{p}_{j}\right\rangle}\left(\omega_{\alpha+\gamma}\right), F_{\left\langle\hat{p}_{j}\right\rangle}\left(\omega_{\alpha}\right)\right) & =\lambda_{j k}(\alpha, \gamma)\left(F_{\left\langle\bar{p}_{k}\right\rangle}\left(\omega_{\alpha+\gamma}\right), F_{\left\langle\bar{p}_{k}\right\rangle}\left(\omega_{\alpha}\right)\right) \\
& \rightarrow \lambda_{j ; l, m}(\alpha, \gamma)\left(Y_{l, m}\left(\omega_{\alpha+\gamma}\right), Y_{l, m}\left(\omega_{\alpha}\right)\right)
\end{aligned}
$$

for some functions $\lambda_{j k}$ and $\lambda_{j ; l, m}$ that may become zero or infinity at finitely many arguments $(\alpha, \gamma)$ only. Taking $j \rightarrow \infty$ leads to

$$
\begin{gathered}
\left(F_{\left\langle\hat{p}_{j}\right\rangle}\left(\omega_{\alpha+\gamma}\right), F_{\left\langle\hat{p}_{j}\right\rangle}\left(\omega_{\alpha}\right)\right)=\lambda_{j ; l, m}(\alpha, \gamma)\left(Y_{l, m}\left(\omega_{\alpha+\gamma}\right), Y_{l, m}\left(\omega_{\alpha}\right)\right) \\
\downarrow \\
\downarrow \\
\left(Y_{l^{\prime}, m^{\prime}}\left(\omega_{\alpha+\gamma}\right), Y_{l^{\prime}, m^{\prime}}\left(\omega_{\alpha}\right)\right)=\lambda_{l^{\prime}, m^{\prime} ; l, m}(\alpha, \gamma)\left(Y_{l, m}\left(\omega_{\alpha+\gamma}\right), Y_{l, m}\left(\omega_{\alpha}\right)\right)
\end{gathered}
$$

for a function $\lambda_{l^{\prime}, m^{\prime} ; l, m}$ that may become zero or infinity at finitely many arguments $(\alpha, \gamma)$ only.

If $\lambda_{l^{\prime}, m^{\prime} ; l, m}(\alpha, \gamma)$ is zero or infinity, then either $Y_{l, m}\left(\omega_{\alpha+\gamma}\right)=0=Y_{l, m}\left(\omega_{\alpha}\right)$ or $Y_{l^{\prime}, m^{\prime}}\left(\omega_{\alpha+\gamma}\right)=0=Y_{l^{\prime}, m^{\prime}}\left(\omega_{\alpha}\right)$, whence the argument $(\alpha, \gamma)$ must be in the set $\left\{(i \pi / \ell, i \pi / \ell): i, j \in \mathbb{Z}, \ell \in\left\{2 l, 2 l^{\prime}\right\}\right\}$. Therefore, the coefficient $\lambda_{i^{\prime}, j^{\prime} ; i, j}(\alpha, \gamma)$ can not be zero or infinity, if both $i$ and $i^{\prime}$ are relative prime to both numbers $2 l$ and $2 l^{\prime}$. Thus, for every pair $(\alpha, \gamma)$ there is an infinite set $\mathcal{L}_{\alpha, \gamma}$ so that $\lambda_{l^{\prime}, m^{\prime} ; l, m}(\alpha, \gamma) \in \mathbb{R} \backslash\{0\}$ for all $m, m^{\prime} \in\{-1,1\}$. If $l^{\prime}, l \in \mathcal{L}_{\alpha, \gamma}$, then (3.20) implies $Y_{l^{\prime}, m^{\prime}}\left(\omega_{\alpha+\gamma}\right) Y_{l, m}\left(\omega_{\alpha}\right)=Y_{l, m}\left(\omega_{\alpha+\gamma}\right) Y_{l^{\prime}, m^{\prime}}\left(\omega_{\alpha}\right)$ for all $m, m^{\prime} \in\{-1,1\}$, which means

$$
\begin{cases}\cos \left(l^{\prime}(\alpha+\gamma)\right) \sin (l \alpha)=\sin (l(\alpha+\gamma)) \cos \left(l^{\prime} \alpha\right), & \text { if }-m=m^{\prime}=1 \\ \sin \left(l^{\prime}(\alpha+\gamma)\right) \cos (l \alpha)=\cos (l(\alpha+\gamma)) \sin \left(l^{\prime} \alpha\right), & \text { if } m=-m^{\prime}=1 \\ \cos \left(l^{\prime}(\alpha+\gamma)\right) \cos (l \alpha)=\cos (l(\alpha+\gamma)) \cos \left(l^{\prime} \alpha\right), & \text { if } m=m^{\prime}=1 \\ \sin \left(l^{\prime}(\alpha+\gamma)\right) \sin (l \alpha)=\sin (l(\alpha+\gamma)) \sin \left(l^{\prime} \alpha\right), & \text { if } m=m^{\prime}=-1\end{cases}
$$

Period.Math. Hungar., $\mathbf{6 7 : 2}$ (2013), 187-209.

(c) Á. Kurusa 
for all $l^{\prime}, l \in \mathcal{L}_{\alpha, \gamma}$. Subtracting the first two equations results in $\sin \left(\left(l^{\prime}-l\right) \alpha+l^{\prime} \gamma\right)=$ $\sin \left(\left(l^{\prime}-l\right) \alpha-l \gamma\right)$, that means either $\left(l^{\prime}+l\right) \gamma \equiv 0(\bmod 2 \pi)$ or $\left(l^{\prime}-l\right)(2 \alpha+\gamma) \equiv$ $\pi(\bmod 2 \pi)$. The latter congruence can not be valid for $l^{\prime}=l$, therefore $2 l \gamma \equiv$ $\pi(\bmod 2 \pi)$ should be valid for every $l \in \mathcal{L}_{\alpha, \gamma}$, which implies $\pi \equiv 2\left(l+l^{\prime}\right) \gamma \equiv$ $2 l \gamma+2 l^{\prime} \gamma \equiv 0(\bmod 2 \pi)$, a contradiction.

Thus, the assumption that system (3.19) degenerates for some $\alpha$ and $\gamma$ was wrong, hence system (3.19) uniquely determines the values $\mu_{\alpha+\beta, r}\left(r y \omega_{\alpha+\beta+\varphi_{1}^{\circ}(y)}\right)$ for every $\alpha$ and $y$. Since the coefficients in system (3.19) depend neither on $r$ nor on $\beta$, the values $\mu_{\alpha+\beta, r}\left(r y \omega_{\alpha+\beta+\varphi_{1}^{\circ}(y)}\right)$ are independent from $r$ and from $\beta$.

The theorem is proved.

Note, that in higher dimensions the symmetry of the conformal Radon transform $\mathrm{R}_{\mathcal{S}, \mu}$ follows from Theorem 3.4, but in the plane we need further conditions.

Theorem 3.5. Let $\mathrm{R}_{\mathcal{S}, \mu}$ be a conformal Radon transform in the plane with symmetric flower $\mathcal{S}$, and let $f \in \mathrm{L}_{*}^{2}\left(\mathbb{R}^{2}\right)$ be of compact support which satisfies condition (1) in Theorem 3.4. Assume further that for each $\ell \in \mathbb{N}$ the function $g(\mathbf{x}):=|\mathbf{x}|^{\ell} f(\mathbf{x})$ satisfies

(2c) $\mathrm{R}_{\mathcal{S}, \mu} g(\Gamma \bar{\omega}, r)=\mathrm{R}_{\mathcal{S}, \mu} g^{\Gamma}(\bar{\omega}, r)$, where $\Gamma$ is the reflection to the $x$-axis. Then $\mu$ is symmetric.

Proof. We use the formulas and notations of the proof of Theorem 3.4. As the flower is symmetric and dilational, there is a function $\varphi(y)$ so that $\varphi_{1}^{ \pm}(y)= \pm \varphi(y)$. Since $\mu$ is conformal, by choosing $\beta=0$ in system (3.19) we obtain

$$
\hat{h}_{\langle\hat{p}\rangle}\left(\omega_{\alpha}, y\right)=F_{\langle\hat{p}\rangle}\left(\omega_{\alpha+\varphi(y)}\right) \mu_{\omega_{0}, 1}\left(y \omega_{\varphi(y)}\right)+F_{\langle\hat{p}\rangle}\left(\omega_{\alpha-\varphi(y)}\right) \mu_{\omega_{0}, 1}\left(y \omega_{-\varphi(y)}\right)
$$

for the function $\hat{h}_{\langle\hat{p}\rangle}\left(\omega_{\alpha}, y\right)=h_{\langle\hat{p}\rangle}^{*}\left(\omega_{\alpha}, y\right) / \sqrt{1+y^{2} \dot{\varphi}^{2}(y)}$. By condition (2c) we also have

$$
\hat{h}_{\langle\hat{p}\rangle}\left(\omega_{\alpha}, y\right)=F_{\langle\hat{p}\rangle}\left(\omega_{\alpha-\varphi(y)}\right) \mu_{\omega_{0}, 1}\left(y \omega_{\varphi(y)}\right)+F_{\langle\hat{p}\rangle}\left(\omega_{\alpha+\varphi(y)}\right) \mu_{\omega_{0}, 1}\left(y \omega_{-\varphi(y)}\right) .
$$

The difference of equations (3.21) and (3.22) implies

$$
0=\sum_{i \in\{-1,1\}}\left(F_{\langle\hat{p}\rangle}\left(\omega_{\alpha+i \varphi(y)}\right)-F_{\langle\hat{p}\rangle}\left(\omega_{\alpha-i \varphi(y)}\right)\right) \mu_{\omega_{0}, 1}\left(y \omega_{i \varphi(y)}\right)
$$

for all $y \in(0,1]$. Substituting the sequence of polynomials $\bar{p}_{k}:=\tilde{p}_{l, m}^{k}(k \in \mathbb{N})$ into (3.23), and taking $k \rightarrow \infty$, by (3.11) we obtain

$$
0=\sum_{i \in\{-1,1\}}\left(Y_{l, m}\left(\omega_{\alpha+i \varphi(y)}\right)-Y_{l, m}\left(\omega_{\alpha-i \varphi(y)}\right)\right) \mu_{\omega_{0}, 1}\left(y \omega_{i \varphi(y)}\right) .
$$

Period.Math. Hungar., 67:2 (2013), 187-209.

(c) Á. Kurusa 
If $Y_{l, m}\left(\omega_{\alpha+\varphi(y)}\right) \neq Y_{l, m}\left(\omega_{\alpha-\varphi(y)}\right)$, then this implies $\mu_{\omega_{0}, 1}\left(y \omega_{\varphi(y)}\right)=\mu_{\omega_{0}, 1}\left(y \omega_{-\varphi(y)}\right)$, which is what we have to prove.

Under the indirect assumption that $Y_{l, m}\left(\omega_{\alpha+\varphi(y)}\right)=Y_{l, m}\left(\omega_{\alpha-\varphi(y)}\right)$ for all $l, m$, considering the special case of $l=1$ and $m= \pm 1$ we get

$$
\begin{aligned}
\cos (\alpha+\varphi(y)) & =\cos (\alpha-\varphi(y)), & & \left(Y_{1,1}\right) \\
\sin (\alpha+\varphi(y)) & =\sin (\alpha-\varphi(y)), & & \left(Y_{1,-1}\right)
\end{aligned}
$$

that implies $\varphi(y)=0$. This can only happen at $y=1$; that proves the theorem.

\section{Identifying conformal Radon transforms}

Thanks to the global structure given by conformality, a complete reconstruction of the flower of a conformal Radon transform* can be given by means of a test function and its image without imposing serious restrictions on the test function. This outcome resembles Mukhometov's results [9], [10], but it is neither a generalization, nor a special case of it.

Observe that one can know a conformal weight without knowing the flower on which it is used, because a conformal weight $\mu$ is determined by a function $\hat{\mu}: \mathbb{R}_{+} \rightarrow \mathbb{R}_{+}$so that $\mu_{\bar{\omega}, r}(\varrho \omega)=\hat{\mu}(\varrho / r)$.

Theorem 4.1. Let $\mathrm{R}_{\mathcal{S}, \mu}$ be a conformal Radon transform, and let $F=\mathrm{R}_{\mathcal{S}, \mu} f$ be known for a known radial function $f \in \mathrm{L}_{*}^{2}\left(\mathbb{R}^{n}\right)$ of compact support that has no vanishing moments. If either the flower or the weight is known and symmetric, then $\mathrm{R}_{\mathcal{S}, \mu}$ is reconstructible by means of $f$ and $F$.

Proof. As $f$ is radial and $\mathrm{R}_{\mathcal{S}, \mu}$ is conformal, $F$ is also radial, whence we have functions $\hat{f}, \hat{F} \in \mathrm{L}_{*}^{2}(\mathbb{R})$ so that $f(\mathbf{x})=\hat{f}(|\mathbf{x}|)$ and $F(\bar{\omega}, r)=\hat{F}(r)$.

Since $\mu$ is a symmetric rotational weight, by $(2.1)$ we have a real function $\bar{\mu}_{r}$ so that $(2.1)$ is fulfilled. Since $\mu$ is also dilational, we have a real function $\bar{\mu}$ for which $\bar{\mu}_{r}(\varrho)=\hat{\mu}(\varrho / r)$.

As $\mathcal{S}$ is rotational and symmetric, equations (2.3) and (2.2) give

$$
F(r)=\mathrm{R}_{\mathcal{S}, \mu} f(\bar{\omega}, r)=\left|\mathbb{S}^{n-2}\right| \int_{0}^{r} \hat{f}(\varrho) \bar{\mu}_{r}(\varrho) \varrho^{n-2} \sin ^{n-2} \varphi_{r}(\varrho) \sqrt{1+\varrho^{2} \dot{\varphi}_{r}^{2}(\varrho)} \mathrm{d} \varrho,
$$

\footnotetext{
* Recall that conformality of a Radon transform can be effectively checked by our Theorems $3.1,3.3$ and 3.4 .
}

Period.Math. Hungar., $\mathbf{6 7 : 2}$ (2013), 187-209.

(c) Á. Kurusa 
where $\bar{\omega}$ is any unit vector and we set $\left|\mathbb{S}^{0}\right|=2$. Since $\mathcal{S}$ and $\mu$ are also dilational, substituting $\varrho=r t$ leads to

$$
r^{1-n} \hat{F}(r)=\left|\mathbb{S}^{n-2}\right| \int_{0}^{1} \hat{f}(r t) \hat{\mu}(t) t^{n-2} \sin ^{n-2} \varphi_{1}(t) \sqrt{1+t^{2} \dot{\varphi}_{1}^{2}(t)} \mathrm{d} t .
$$

Integration over $[0, \infty]$ by $r^{n-1-k} \mathrm{~d} r(2 \leq k \in \mathbb{N})$ results in

$$
\begin{aligned}
& \int_{0}^{\infty} r^{-k} \hat{F}(r) \mathrm{d} r \\
& \quad=\left|\mathbb{S}^{n-2}\right| \int_{0}^{\infty} \varrho^{n-1-k} \hat{f}(\varrho) \mathrm{d} \varrho \int_{0}^{1} \hat{\mu}(t) t^{k-2} \sin ^{n-2} \varphi_{1}(t) \sqrt{1+t^{2} \dot{\varphi}_{1}^{2}(t)} \mathrm{d} t .
\end{aligned}
$$

By the Stone-Weierstrass Theorem the set of functions $t^{k-2}(2 \leq k \in \mathbb{N})$ is a complete base in the set $\mathrm{L}^{2}[0,1]$, whence equation (4.1) determines the function

$$
\psi(t)=\hat{\mu}(t) \sin ^{n-2} \varphi_{1}(t) \sqrt{1+t^{2} \dot{\varphi}_{1}^{2}(t)} .
$$

If $\mathcal{S}$ is known, then equation (4.2) determines $\hat{\mu}(t)$, that proves the statement.

If $\mu$ is known, then (4.2) determines $\bar{\psi}(t)=\sin ^{n-2} \varphi_{1}(t) \sqrt{1+t^{2} \dot{\varphi}_{1}^{2}(t)}$. This is a non-linear ordinary differential equation for $\varphi_{1}$ with boundary conditions $\varphi_{1}(1)=$ 0 and $\varphi_{1}(\varrho)(1-\varrho)^{-1 / 2} \in \mathrm{C}^{\left[\frac{n+2}{2}\right]}([0,1])$, therefore the Pickard-Lindelöf Theorem implies the uniqueness of its solution, that proves the statement.

A differentiable conformal flower is said to be selftangent if it has two tangent petals. Clearly, a flower is selftangent if and only if its generating curve $c_{r}$ has two points where the respective tangents of $c_{r}$ close supplementary signed angles to their respective radial lines. Furthermore, a flower is selftangent if and only if its dual flower is selftangent too, because

$$
\begin{aligned}
\dot{c}_{r}(\varrho) & =\frac{\partial}{\partial \varrho}\left(\varrho\left(\cos \varphi_{r}(\varrho), \sin \varphi_{r}(\varrho)\right)\right) \\
& =\left(\cos \varphi_{r}(\varrho), \sin \varphi_{r}(\varrho)\right)+\varrho\left(-\sin \varphi_{r}(\varrho), \cos \varphi_{r}(\varrho)\right) \dot{\varphi}_{r}(\varrho)
\end{aligned}
$$

means that $\tan (\alpha(\varrho))=\varrho \dot{\varphi}_{r}(\varrho)=r \dot{\psi}_{\varrho}(r)$, where $\alpha(\varrho)$ is the signed angle that the radial line through $c_{r}(\varrho)$ closes to the tangent $\dot{c}_{r}(\varrho)$ at the point $c_{r}(\varrho)$. These imply immediately that the flower $\mathcal{H}$ of the hyperplanes and its dual flower, the set of the spheres through the origin, are not selftangent.

Our next Theorem 4.2 generalizes and sharpens the results of Boman [1] and Natterer [11], [12].

Period.Math. Hungar., $\mathbf{6 7 : 2}$ (2013), 187-209.

(c) Á. Kurusa 
Theorem 4.2. Let the differentiable conformal flower $\mathcal{S}$ be not selftangent. If $\mathrm{R}_{\mathcal{S}, \mu} f(\bar{\omega}, r)=F(\bar{\omega}, r)$ is known for an unknown function $f=\sum_{i=1}^{m} \delta_{\mathbf{x}_{i}}+\ell$, where $\ell \in \mathrm{L}^{2}\left(\mathbb{R}^{n}\right)$ and $\delta_{\mathbf{x}_{i}}$ is the Dirac measure at $\mathbf{x}_{i} \in \mathbb{R}^{n} \backslash\{\mathbf{0}\}$, then the generating curve $c_{1}$ is determined, and the weights $\mu_{\omega, r}\left(\mathbf{x}_{i}\right)$ can be calculated for the pairs $(\omega, r) \in \mathbb{S}^{n-1} \times \mathbb{R}_{+}$satisfying $\mathbf{x}_{i} \in S_{\omega, r}$.

Proof. Consider the sequence of functions

$$
g_{\mathbf{x}, j}(\mathbf{y})= \begin{cases}0, & \text { if }|y-x|>1 / j \\ j^{n-1}, & \text { if }|y-x|<1 / j .\end{cases}
$$

As $\mathrm{R}_{\mathcal{S}, \mu}^{*}=\mathrm{R}_{\mathcal{S}^{*}, \mu^{*}}$ with $\mathcal{S}^{*}$ and $\mu^{*}$ defined in Theorem 2.2 , we clearly have

$$
\lim _{j \rightarrow \infty}\left\langle\ell, \mathrm{R}_{\mathcal{S}^{*}, \mu^{*}} g_{\mathbf{x}, j}\right\rangle=\lim _{j \rightarrow \infty}\left\langle\mathrm{R}_{\mathcal{S}, \mu} \ell, g_{\mathbf{x}, j}\right\rangle=0
$$

for all $\mathbf{x} \in \mathbb{R}^{n}$, hence

$$
\lim _{j \rightarrow \infty}\left\langle\mathrm{R}_{\mathcal{S}, \mu} f, g_{\mathbf{x}, j}\right\rangle=\lim _{j \rightarrow \infty}\left\langle\sum_{i=1}^{m} \delta_{\mathbf{x}_{i}}+\ell, \mathrm{R}_{\mathcal{S}^{*}, \mu^{*}} g_{\mathbf{x}, j}\right\rangle=\sum_{i=1}^{m} \lim _{j \rightarrow \infty} \mathrm{R}_{\mathcal{S}^{*}, \mu^{*}} g_{\mathbf{x}, j}\left(\mathbf{x}_{i}\right) .
$$

The $\operatorname{limit}_{\lim _{j \rightarrow \infty}} \mathrm{R}_{\mathcal{S}^{*}, \mu^{*}} g_{\mathbf{x}, j}\left(\mathbf{x}_{i}\right)$ is not zero if and only if $\mathbf{x} \in S_{\omega_{i}, r_{i}}^{*}$. Therefore the set $\hat{\mathcal{S}}:=\bigcup_{i=1}^{m} S_{\omega_{i}, r_{i}}^{*}$ is the support of the function $h(\mathbf{x}):=\lim _{j \rightarrow \infty}\left\langle\mathrm{R}_{\mathcal{S}, \mu} f, g_{\mathbf{x}, j}\right\rangle$.

As the flower $\mathcal{S}$ is not selftangent, its dual flower $\mathcal{S}^{*}$ is not selftangent too, therefore no petal $S_{\omega_{i}, r_{i}}^{*}(i=1, \ldots, m)$ can be tangent to any other petal $S_{\omega_{j}, r_{j}}^{*}$ $(j=1, \ldots, m)$ if $i \neq j$, therefore each point of $\hat{\mathcal{S}}$ has a neighbourhood in which one can decide if two points of $\hat{\mathcal{S}}$ belong to the same petal $S_{\omega_{i}, r_{i}}^{*}$ for a suitable $i=$ $1, \ldots, m$ or not. This is enough to determine the petals $S_{\omega_{i}, r_{i}}^{*}$ for all $i=1, \ldots, m$, hence we get $c_{\omega_{i}, r_{i}}^{*}$ and therefore we have $c_{\omega_{i}, r_{i}}$.

Having the petals, one can calculate the weight for all $\mathrm{x} \in S_{\omega_{i}, r_{i}}^{*} \backslash \hat{\mathcal{S}}$ by

$$
\begin{aligned}
\lim _{j \rightarrow \infty} \frac{\left\langle F, g_{\mathbf{x}, j}\right\rangle\left|\mathbf{x}_{i}\right| /|\mathbf{x}|}{\mathrm{R}_{\mathcal{S}^{*}, 1} g_{\mathbf{x}, j}\left(\mathbf{x}_{i}\right)} & =\lim _{j \rightarrow \infty} \frac{\left|\mathbf{x}_{i}\right|\left\langle\mathrm{R}_{\mathcal{S}, \mu} f, g_{\mathbf{x}, j}\right\rangle}{|\mathbf{x}| \mathrm{R}_{\mathcal{S}^{*}, 1} g_{\mathbf{x}, j}\left(\mathbf{x}_{i}\right)}=\lim _{j \rightarrow \infty} \frac{\left|\mathbf{x}_{i}\right| \mathrm{R}_{\mathcal{S}^{*}, \mu^{*}} g_{\mathbf{x}, j}\left(\mathbf{x}_{i}\right)}{|\mathbf{x}| \mathrm{R}_{\mathcal{S}^{*}, 1} g_{\mathbf{x}, j}\left(\mathbf{x}_{i}\right)} \\
& =\frac{\left|\mathbf{x}_{i}\right|}{|\mathbf{x}|} \mu_{\omega_{i}, r_{i}}^{*}(\mathbf{x})=\mu_{\mathbf{x} /|\mathbf{x}|,|\mathbf{x}|}\left(\mathbf{x}_{i}\right) .
\end{aligned}
$$

In the intersections of the petals the weight can be determined by continuity; that completes the proof. 
Note that after $\mathcal{S}$ and $\mu$ become known, the noise ${ }^{\dagger} \ell$ can also be determined with the inversion formulas in [7] and [13] provided $\mu$ is rotation invariant.

Much better results than Theorem 4.2 can hardly be reached in general, because $\mathrm{R}_{\mathcal{S}, \mu} f$ can not serve information about the weight on those petals that do not intersect the support of $f$ unless the weight has a global structure as in the following theorem.

Theorem 4.3. Let the differentiable flower $\mathcal{S}$ be conformal and not selftangent. Assume, that for a continuous function $\nu: \mathbb{R}_{+}^{2} \rightarrow \mathbb{R}$, which is strictly monotone in its second variable, the weight $\hat{\mu}_{\omega, r}(\mathbf{x}):=\nu\left(r, \mu_{\omega, r}(\mathbf{x})\right)$ is conformal.

If $\mathrm{R}_{\mathcal{S}, \mu} f(\bar{\omega}, r)=F(\bar{\omega}, r)$ is known for an unknown function $f=\sum_{i=1}^{m} \delta_{\mathbf{x}_{i}}+\ell$, where $\ell \in \mathrm{L}^{2}\left(\mathbb{R}^{n}\right)$ and $\delta_{\mathbf{x}_{i}}$ is the Dirac measure at $\mathbf{x}_{i} \in \mathbb{R}^{n} \backslash\{\mathbf{0}\}$, then the generating curve $c_{1}$ and the weight $\mu$ can be calculated.

Proof. Since $\hat{\mu}$ is conformal, by (2.1) we have

$$
\nu\left(r, \mu_{\omega, r}(\mathbf{x})\right)=\hat{\mu}_{\omega, r}(\mathbf{x})= \begin{cases}\bar{\mu}\left(\frac{|\mathbf{x}|}{r} \frac{\left\langle\mathbf{x}, \omega^{\perp}\right\rangle}{\left|\left\langle\mathbf{x}, \omega^{\perp}\right\rangle\right|}\right), & \text { if } n=2, \\ \bar{\mu}(|\mathbf{x}| / r), & \text { if } n>2,\end{cases}
$$

for a suitable real function $\bar{\mu}$ defined either on $[-1,1]$ or $[0,1]$.

As the flower $\mathcal{S}$ is rotational, for any $\mathbf{x}_{i}$ and $r \geq\left|\mathbf{x}_{i}\right|$ there is an $\omega \in \mathbb{S}^{n-1}$ so that $\mathbf{x}_{i} \in S_{\omega, r}$, whence we can define a function $\omega_{i}: \mathbb{R}_{+} \rightarrow \mathbb{S}^{n-1}$ so that $\mathbf{x}_{i} \in S_{\omega_{i}(r), r}$. This means by Theorem 4.2 that $\nu\left(r, \mu_{\omega_{i}(r), r}\left(\mathbf{x}_{i}\right)\right)$ can be calculated for all $r \geq\left|\mathbf{x}_{i}\right|$, which determine $\bar{\mu}$ via (4.3) on its domain.

Let $\bar{\nu}_{r}$ denote the inverse of the function $\nu_{r}:=\nu(r, \cdot)$. Then

$$
\mu_{\omega, r}(\mathbf{x})=\bar{\nu}_{r}\left(\hat{\mu}_{\omega, r}(\mathbf{x})\right)= \begin{cases}\bar{\nu}_{r}\left(\bar{\mu}\left(\frac{|\mathbf{x}|}{r} \frac{\left\langle\mathbf{x}, \omega^{\perp}\right\rangle}{\left|\left\langle\mathbf{x}, \omega^{\perp}\right\rangle\right|}\right)\right), & \text { if } n=2, \\ \bar{\nu}_{r}(\bar{\mu}(|\mathbf{x}| / r)), & \text { if } n>2,\end{cases}
$$

proves the statement.

Note that our result covers the Radon transforms with exponential weight $\mu_{\omega, r}(\mathbf{x}):=e^{a|\mathbf{x}-r \omega|}=e^{a \sqrt{|\mathbf{x}|^{2}-r^{2}}}=\left(e^{a \sqrt{(|\mathbf{x}| / r)^{2}-1}}\right)^{r}$, therefore generalizes Solmon's result [16] for more general flowers, but using much more restricted test functions.

$\left\lceil\right.$ We call the added $\mathrm{L}^{2}$-function noise.

Period.Math. Hungar., 67:2 (2013), 187-209.

(c) Á. Kurusa 


\section{Appendix of auxiliary concepts}

We need the following facts about spherical harmonics: A complete orthonormal system in the Hilbert space $\mathrm{L}^{2}\left(\mathbb{S}^{n-1}\right)$ can be chosen consisting of spherical harmonics $Y_{l, m}$, where $Y_{l, m}$ is of degree $l$ and $m$ has $(2 l+n-2)(l+n-3) ! / l !(n-2)$ ! different values. Having such a system, $f \in \mathrm{C}^{\infty}\left(\mathbb{S}^{n-1} \times[0, \infty)\right), p \in[0, \infty)$ and $f_{l, m}(q)=\int_{\mathbb{S}^{n-1}} f(q \omega) Y_{l, m}(\omega) \mathrm{d} \omega$, then the series

$$
\sum_{l, m}^{\infty} f_{l, m}(p) Y_{l, m}(\omega):=\sum_{l=1}^{\infty} \sum_{m} f_{l, m}(p) Y_{l, m}(\omega)
$$

converges uniformly absolutely on any compact subset of $\mathbb{S}^{n-1} \times[0, \infty)$ to $f(p \omega)$. On $\mathbb{S}^{1}$ we choose $Y_{0,0}\left(\omega_{\alpha}\right)=1 / 2 \pi$, and $Y_{l, 1}\left(\omega_{\alpha}\right)=\cos (l \alpha) / \pi$ and $Y_{l,-1}\left(\omega_{\alpha}\right)=\sin (l \alpha) / \pi$ for $0<l \in \mathbb{N}$. For further details on spherical harmonics we refer to [15].

We need some facts about the Pompeiu property. We say that a function $f \in \mathrm{L}^{2}\left(\mathbb{S}^{n-1}\right)$ has the Pompeiu property, if the only weight $\mu \in C\left(\mathbb{S}^{n-1}\right)$ satisfying

$$
P_{f} \mu(\Phi):=\int_{\mathbb{S}^{n-1}} \mu(\omega) f(\Phi \omega) \mathrm{d} \omega \equiv 0
$$

for every $\Phi \in S O(n)$ is $\mu \equiv 0$. Accordingly, a domain on the sphere is said to have the Pompeiu property, if its characteristic function has the Pompeiu property. For $n=2$, an arc has the Pompeiu property if and only if its length is incommensurable to $\pi$ [17]. The generalizations of this for the cases $n=3$ and for $n \geq 3$, are due to Ungar [18, Freak Theorem] and Schneider [14, Korollar 3.3], respectively. In sum we can say that the respective sets of angles $\alpha \in(0, \pi / 2)$ for which $\alpha$-caps ${ }^{(5: 2)}$ have or fail the Pompeiu property are everywhere dense, respectively.

We say that a function $f \in \mathrm{L}^{2}\left(\mathbb{S}^{n-1}\right)$ has the attenuated hyper Pompeiu property $([8])$ if there is an everywhere dense set $\mathcal{G}$ of great spheres of $\mathbb{S}^{n-1}$ so that the restriction of $f$ on any great sphere in $\mathcal{G}$ has the Pompeiu property.

There is no function on $\mathbb{S}^{1}$ with the attenuated hyper Pompeiu property, but for higher dimensions the characteristic function of any $\alpha$-sector ${ }^{(5: 3)}$ has the attenuated hyper Pompeiu property.

(5:2) An $\alpha$-cap is the set of common points of the sphere with a right circular cone of aperture $2 \alpha$ with apex in the centre of the sphere.

(5:3) An $\alpha$-sector of a sphere is a region of the sphere between two great spheres intersecting at an angle $\alpha$. In dimension 2 it is a spherical bi-angle with angle $\alpha$.

Period.Math. Hungar., 67:2 (2013), 187-209.

(c) Á. Kurusa 
Theorem 5.1. The indicator function of an $\alpha$-sector on $\mathbb{S}^{n-1}(n \geq 3)$ has the attenuated hyper Pompeiu property.

Proof. Let $\mathcal{A}$ be an $\alpha$-sector on $\mathbb{S}^{n-1}$, and let $\mathcal{S}$ be any great sphere on $\mathbb{S}^{n-1}$.

The intersection $\mathcal{B}:=\mathcal{A} \cap \mathcal{S}$ is a $\beta$-cap on $\mathcal{S}$.

If $\mathcal{S}$ does not contain the vertices of $\mathcal{A}$, then $\beta$ is in $(\alpha, \pi / 2)$ and it changes continuously with respect to any movement of $\mathcal{S}$ in a small neighbourhood, therefore $\mathcal{B}$ has the Pompeiu property for some great sphere in any neighbourhood of $\mathcal{S}$.

If $\mathcal{S}$ contains the vertices of $\mathcal{A}$, then $\beta=0$ or $\beta=\pi / 2$, and in any neighbourhood of $\mathcal{S}$ there is a connected open set of great spheres $\mathcal{S}^{\prime}$, that do not contain the vertices of $\mathcal{A}$. By the previous paragraph, in any neighbourhood of $\mathcal{S}^{\prime}$ there is a great sphere $\mathcal{S}^{\prime \prime}$ on which $\mathcal{B}^{\prime \prime}:=\mathcal{A} \cap \mathcal{S}^{\prime \prime}$ has the Pompeiu property.

The proof is complete.

\section{References}

[1] J. Boman, On generalized Radon transforms with unknown measures, Integral geometry and tomography (Arcata, CA, 1989), Contemp. Math. 113, Amer. Math. Soc., Providence, RI, 1990, 5-15.

[2] A. M. Cormack and E. T. Quinto, A Radon transform on spheres through the origin in $\mathbb{R}^{n}$, Trans. Amer. Math. Soc., 260 (1980), 575-581.

[3] A. M. Cormack, The Radon transform on a family of curves in the plane I-II, Proc. Amer. Math. Soc., 83;86 (1981;1982), 325-330; 293-298.

[4] A. Hertle, A characterization of Fourier and Radon transforms on Euclidean space, Trans. Amer. Math. Soc., 273 (1982), 595-607.

[5] A. Hertue, The identification problem for the constantly attenuated Radon transform, Math. Z., 197 (1988), 13-19.

[6] Á. Kurusa, Characterization of the Radon transform and its dual on Euclidean space, Acta Sci. Math. (Szeged), 54 (1990), 273-276.

[7] Á. Kurusa, Support curves of invertible Radon transforms, Arch. Math., 61 (1993), 448-458.

[8] Á. Kurusa, Hyper Pompeiu property (2011), manuscript.

[9] R. G. Muкнометоv, The reconstruction problem of a two-dimensional Riemannian metric and integral geometry, Sov. Math. Dokl., 18 (1977), 27-31.

[10] R. G. Muknometov, A problem of reconstructing a Riemannian metric, Sib. Math. J., 22 (1981), 420-433.

[11] F. Natterer, Computerized tomography with unknown sources, SIAM J. Appl. Math., 43 (1983), 1201-1212.

[12] F. Natterer, An inverse problem for a transport equation and integral geometry, Contemp. Math., 113 (1990), 221-231. 
[13] E. T. Quinto, The invertibility of rotation invariant Radon transforms, Math. Anal. Appl., 91 (1983), 510-522.

[14] R. Schneider, Über eine Integralgleichung in der Theorie der konvexen Körper, Math. Nachr., 44 (1970), 55-74.

[15] R. Seeley, Spherical harmonics, Amer. Math. Monthly, 73 (1966), 115-121.

[16] D. C. Solmon, The identification problem for the exponential Radon transform, Math. Methods Appl. Sci., 18:9 (1995), 687-695.

[17] D. C. Ulrich, More on the Pompeiu Problem, Amer. Math. Monthly, 101 (1994), $165-168$.

[18] P. Ungar, Freak theorem about functions on a sphere, J. London Math. Soc.,

29 (1954), 100-103.

Á. Kurusa, Bolyai Institute, Aradi vértanúk tere 1., H-6720 Szeged, Hungary; e-mail: kurusa@math.u-szeged.hu 